\title{
Piano Stool Aminoalkylidene-Ferracyclopentenone Complexes from Bimetallic Precursors: Synthesis and Cytotoxicity Data
}

Dalila Rocco, ${ }^{\mathrm{a}, \S}$ Dr. Lucinda K. Batchelor, ${ }^{\mathrm{b}}$ Dr. Eleonora Ferretti, ${ }^{\mathrm{c}, \#}$ Prof. Stefano Zacchini, ${ }^{\mathrm{d},{ }^{*}}$ Prof. Guido Pampaloni, ${ }^{\text {a }}$ Prof. Paul J. Dyson, ${ }^{\mathrm{b}}$ Prof. Fabio Marchetti ${ }^{\mathrm{a},{ }^{*}}$

\footnotetext{
${ }^{a}$ Dipartimento di Chimica e Chimica Industriale, Università di Pisa, Via G. Moruzzi 13, I-56124 Pisa, Italy.

${ }^{b}$ Institut des Sciences et Ingénierie Chimiques, Ecole Polytechnique Fédérale de Lausanne (EPFL), CH-1015 Lausanne, Switzerland.

${ }^{c}$ Institut für Anorganische Chemie, Tammannstr. 4, D-37077 Göttingen, Germany

${ }^{d}$ Dipartimento di Chimica Industriale "Toso Montanari”, Università di Bologna, Viale Risorgimento 4, I-40136 Bologna, Italy.
}

\section{Corresponding Authors}

*E-mail addresses:

fabio.marchetti1974@unipi.it. Webpage: http://people.unipi.it/fabio_marchetti1974/ stefano.zacchini@unibo.it

Present addresses: ${ }^{\S}$ Department of Chemistry, University of Basel, BPR 1096, Mattenstrasse 24a, CH4058 Basel, Switzerland; " Institut für Chemie und Biochemie, Fabeckstr 34-36, 14195 Berlin. 


\section{Table of contents}

Amine-promoted selective fragmentation of readily available diiron vinyliminium complexes can be broadly applied to the synthesis of piano-stool monoiron products featuring a unique structural motif, which is partially constructed by exploiting Fe-Fe cooperativity. The compounds display a variable cytotoxicity against cancer (A2780 and A280cisR) and non-cancerous (HEK293) cell lines, and a significant selectivity has been observed with a phenyl ring as vinyl substituent.

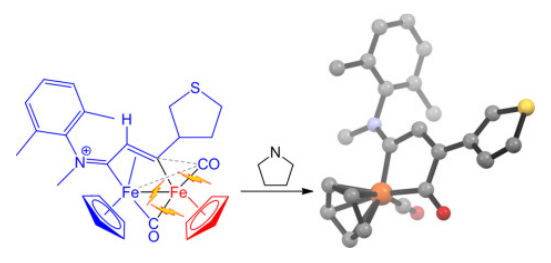




\begin{abstract}
The reaction of pyrrolidine with a series of cationic diiron cyclopentadienyl complexes containing a bridging vinyliminium ligand gives access to piano stool monoiron complexes based on a fivemembered metallacycle that includes a vinyl-aminoalkylidene moiety, in moderate to high yields. The resulting metallacyclic motif (aminoalkylidene-ferracyclopentenone) is unique in organometallic chemistry, and is partially pre-constructed on the dinuclear frame. The monoiron products were fully characterized by elemental analysis, IR and multinuclear NMR spectroscopy, and in a number of cases by X-ray diffraction and cyclic voltammetry. They are robust in aqueous solutions and generally unreactive towards alkylating agents in organic solvents. However, a cationic derivative was prepared in high yield by methylation of a 2-pyridyl group. The cytotoxicity of both neutral and ionic complexes was assessed on cancer (A2780 and A280cisR) and non-cancerous (HEK293) cell lines, revealing the influence of local structural modifications on the antiproliferative activity and the selectivity of the compounds.
\end{abstract}

Keywords: cytotoxicity; iron; metal-based drugs; piano stool complexes; vinyliminium ligands.

\title{
Introduction
}

Interest in developing new iron-based compounds is motivated by the attractive characteristics of the metal, being abundant, inexpensive and environmentally benign. ${ }^{[1]}$ In particular, piano-stool iron complexes containing the robust cyclopentadienyl (Cp) ligand have been intensively investigated for their catalytic applications, ${ }^{[2]}$ and, following the interest aroused by the anticancer potential of ferrocene derivatives, ${ }^{[3]}$ also as cytotoxic agents. ${ }^{[4]}$ In general, such class of compounds may be obtained from commercially available $\mathrm{Fe}_{2} \mathrm{Cp}_{2}(\mathrm{CO})_{4}$ or its simple derivatives, via preliminary fragmentation of the dinuclear frame. Thus, the smooth oxidation of $\mathrm{Fe}_{2} \mathrm{Cp}_{2}(\mathrm{CO})_{4}$ by means of either 
halogen-based oxidants (e.g. hydrogen halides ${ }^{[5]}$ and $\mathrm{I}_{2}{ }^{[6]}$ ) or silver salts ${ }^{[7]}$ provides a facile entry into mononuclear species based on the $\left[\mathrm{FeCp}(\mathrm{CO})_{2}\right]^{+}$core, to which a variety of ligands can be added. ${ }^{\text {[2a- }}$ b,d,4a,6,8] Small molecular units tethered to the $\left[\mathrm{Fe}_{2} \mathrm{Cp}_{2}(\mathrm{CO})_{3}\right]$ framework prior to fragmentation may be preserved in the corresponding monoiron products, and examples include isocyanides, ${ }^{[9]}$ thiocarbonyl [10] and vinyl groups. ${ }^{[1]}$ Alternative strategies for the synthesis of piano stool iron(II) carbonyl compounds are constituted by $\mathrm{Na}(\mathrm{Hg})$ reduction of $\mathrm{Fe}_{2} \mathrm{Cp}_{2}(\mathrm{CO})_{4}{ }^{[12]}$ and the use of iron(II) chloride/sodium cyclopentadienide systems. ${ }^{[2 \mathrm{c}]}$ In general, Fischer alkylidene (Fischer carbene) ligands, including aminoalkylidene ligands, need to be built directly on monoiron complexes via modification of hydrocarbyl ligands, thus determining intrinsic structural limitations. ${ }^{[13]}$ In this regard, (Cp)Feaminocarbene moieties have been obtained by aminolysis of alkoxy-carbenes ${ }^{[14]}$ and vinylidenes, ${ }^{[15]}$ modification of isocyanide ligands, ${ }^{[14 a]}$ and electrophilic addition of imidoyl chlorides to suitable organoferrate(I) anions. ${ }^{[16]}$

A more versatile approach would consist in the construction of a functionalized ligand on the diiron scaffold $\mathrm{Fe}_{2} \mathrm{Cp}_{2}(\mathrm{CO})_{\mathrm{x}}(\mathrm{x}=2-3)$, followed by cleavage of the latter and subsequent inclusion of the preformed fragment in a monoiron compound. In principle, this strategy takes advantage of the cooperative effects provided by the two adjacent iron centres, exploiting bridging and multisite coordination modes and allowing reactivity patterns which are not available on homologous mononuclear structures. ${ }^{[17]}$

Diiron complexes with a bridging vinyliminium ligand, 2, can be prepared on gram scales from $\mathrm{Fe}_{2} \mathrm{Cp}_{2}(\mathrm{CO})_{4}$ through the stepwise assembly of one isocyanide and one alkyne, proceeding with the intermediate formation of the $\mu$-aminocarbyne species 1 (Scheme 1$).{ }^{[18,19]}$ The diiron compounds 2 display notable properties, including promising antiproliferative potential, ${ }^{[20]}$ and their versatile chemistry offers much opportunity for unusual and selective transformations of the vinyliminium moiety; ${ }^{[17 a, 21]}$ in general, the resulting organic fragments remain anchored to the two iron atoms. Herein, we describe the facile synthesis (tolerant of various functionalities) of a large family of piano 
stool monoiron compounds incorporating the $\mathrm{N}-\mathrm{C}^{1}-\mathrm{C}^{2}-\mathrm{C}^{3}$ moiety and also report on their antiproliferative potential.

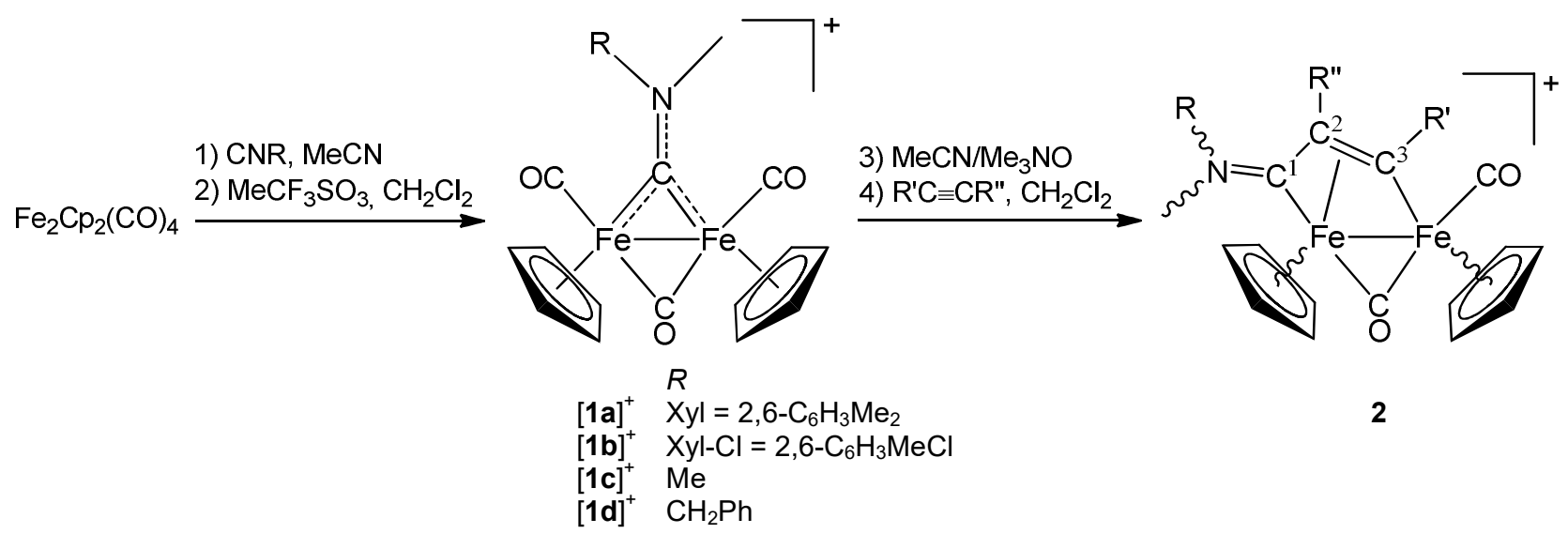

Scheme 1. Synthesis of diiron $\mu$-vinyliminium complexes (counteranion $=\mathrm{CF}_{3} \mathrm{SO}_{3}{ }^{-} ; \mathrm{R}^{\prime}=\mathrm{H}$, alkyl, aryl, $\mathrm{SiMe}_{3}$, $\mathrm{CO}_{2} \mathrm{Me}, \mathrm{N}$ - or S-heterocycle; $\mathrm{R}^{\prime \prime}=\mathrm{H}$, alkyl or aryl).

\section{Results and discussion}

\section{Synthesis and characterization of compounds.}

A series of vinyliminium compounds, $[\mathbf{2 a}-\mathbf{m}] \mathrm{CF}_{3} \mathrm{SO}_{3}$, was prepared as air stable triflate salts in $60-90 \%$ yield from the parent aminocarbyne complexes, following a literature procedure (Scheme 1). ${ }^{20}$ Complexes [2 $\mathbf{b}, \mathbf{d}, \mathbf{g}, \mathbf{i}, \mathbf{j}, \mathbf{l}] \mathrm{CF}_{3} \mathrm{SO}_{3}$ are unprecedented, and their IR and NMR features generally resemble those typical of related species bearing a cis geometry of the Cp ligands and $E$ arrangement of the Nsubstituents (when $\mathrm{R} \neq \mathrm{Me}$ ). The NMR spectra of $[\mathbf{2} \mathbf{d}] \mathrm{CF}_{3} \mathrm{SO}_{3}$ and $[\mathbf{2} \mathbf{j}] \mathrm{CF}_{3} \mathrm{SO}_{3}$ (in acetone-d $\mathrm{d}^{6}$ ) contain two sets of resonances, attributed to $\mathrm{E} / \mathrm{Z}$ and trans/cis isomers, respectively. Complex $[\mathbf{2 g}] \mathrm{CF}_{3} \mathrm{SO}_{3}$ also exists in solution as a mixture of two species, differing in the spatial orientation of the $\mathrm{Me}$ and $\mathrm{Cl}$ substituents on the N-bound arene. The reaction involving $[\mathbf{1 c}] \mathrm{CF}_{3} \mathrm{SO}_{3}$ and 2-ethynylpyridine was not selective, and two geometric isomers were obtained (head-head and head-tail alkyne insertion modes), as evidenced by NMR spectroscopy, see Chart 7. The structures of $[\mathbf{2 i}] \mathrm{CF}_{3} \mathrm{SO}_{3}$ and trans- $[\mathbf{2} \mathbf{j}] \mathrm{CF}_{3} \mathrm{SO}_{3}$ 
were ascertained by single crystal X-ray diffraction (views of the structures are provided as Supporting Information, see Figures S1 and S2).

We recently described the cobaltocene-induced fragmentation of some diiron vinyliminium compounds, leading to the monoiron $\left[\mathrm{FeCp}(\mathrm{CO})\left\{\mathrm{C}^{1} \mathrm{~N}(\mathrm{Me})(\mathrm{Xyl}) \mathrm{C}^{2} \mathrm{HC}^{3}\left(\mathrm{R}^{\prime}\right) \mathrm{C}(=\mathrm{O})\right\}\right]\left(\mathrm{R}^{\prime}=\mathrm{Ph}, \mathbf{3 a} ; \mathrm{R}^{\prime}=\right.$ $\left.\mathrm{CH}_{2} \mathrm{OH}, 3 \mathbf{3 n} ; \mathrm{R}^{\prime}=\mathrm{Et}, \mathbf{3 0}\right) \cdot{ }^{[22,20]} \mathrm{A}$ detailed investigation on the synthetic pathway to $3 \mathbf{o}$ indicated that initial single electron transfer from $\mathrm{CoCp}_{2}$ to the diiron complex triggers a multistep rearrangement, terminating with the elimination of one iron atom and cyclopentadiene. ${ }^{[22]}$ These reactions require rigorously anhydrous conditions: $\mathrm{CoCp}_{2}$ is air/moisture sensitive, therefore the reaction solvent must be freshly distilled and, since the diiron reactant is hydrophilic, this needs to be stored under inert atmosphere or dried before use. Furthermore, the reactions seem limited to specific R' substituents. On the other hand, the employment of a stronger reductant such as sodium hydride, that is also an efficient Brönsted base, may favour deprotonation pathways. ${ }^{[23]}$

In order to find a convenient and general method to obtain compounds of type $\mathbf{3}$, we considered the possibility of using an amine in the place of cobaltocene; the reducing power of amines is well documented in the literature. ${ }^{[2]}$ To this purpose, pyrrolidine was selected as the optimal reagent, due to its favourable characteristics (liquid at ambient temperature, relatively low boiling point and inexpensive); instead the use of other amines was less satisfying $\left(\mathrm{NH}_{2} \mathrm{Cy}, \mathrm{NH}_{2} \mathrm{Et}\right)$, or unsuccessful $\left(\mathrm{NEt}_{3}, \mathrm{NH}_{2}{ }^{\mathrm{i}} \mathrm{Pr}\right)$. Thus, the reactions of $\mathbf{2} \mathbf{a}-\mathbf{m}$ with a ten-fold excess of pyrrolidine in tetrahydrofuran afforded 3a-m in 40-93\% yields (referred to the $C^{1}-C^{3}$ chain); no significant amounts of side-products were detected in all cases. The method reported in Scheme 2 is not critically sensitive to air/moisture (pyrrolidine is used from the bottle and 2a-m can be conserved in air without any pre-treatment before reaction) and, notably, can be broadly applied as it tolerates a variety of functional groups. 


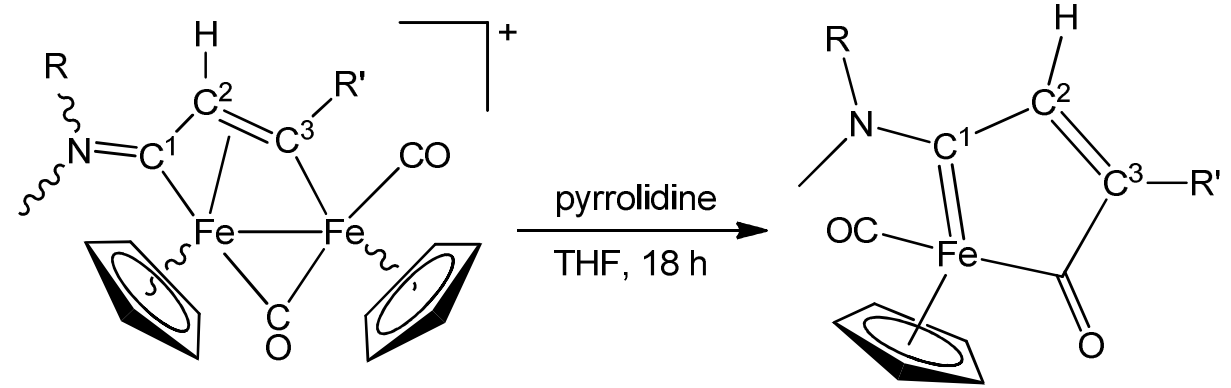

\begin{tabular}{|c|c|c|c|}
\hline & $R$ & $R^{\prime}$ & \\
\hline$[2 \mathrm{a}]^{+}$ & Xyl & $\mathrm{Ph}$ & $3 a$ \\
\hline$[2 b]^{+}$ & Xyl & ${ }^{\mathrm{t} B u}$ & $3 b$ \\
\hline$[2 c]^{+}$ & Xyl & 2-naphthyl & 3c \\
\hline$[\mathbf{2 d}]^{+}$ & $\mathrm{Xyl}$ & 3-pyridyl & 3d \\
\hline$[2 e]^{+}$ & Xyl & 2-pyridyl & $3 e$ \\
\hline$[2 \mathrm{f}]^{+}$ & Xyl & 3-thiophenyl & $3 f$ \\
\hline$[2 \mathbf{g}]^{+}$ & Xyl-Cl & $\mathrm{Ph}$ & $3 \mathbf{g}$ \\
\hline$[2 \mathrm{~h}]^{+}$ & $\mathrm{Me}$ & $\mathrm{Ph}$ & $3 \mathrm{~h}$ \\
\hline$[2 i]^{+}$ & $\mathrm{Me}$ & $3,5-\mathrm{C}_{6} \mathrm{H}_{3}\left(\mathrm{CF}_{3}\right)_{2}$ & $3 \mathbf{i}$ \\
\hline$[2 \mathrm{j}]^{+}$ & $\mathrm{Me}$ & ${ }^{\mathrm{t}} \mathrm{Bu}$ & $3 \mathbf{j}$ \\
\hline$[\mathbf{2 k}]^{+}$ & $\mathrm{Me}$ & 2-naphthyl & $3 \mathbf{k}$ \\
\hline$[21]^{+}$ & $\mathrm{Me}$ & 3-pyridyl & 31 \\
\hline$[2 \mathrm{~m}]^{+}$ & $\mathrm{CH}_{2} \mathrm{Ph}$ & $\mathrm{Ph}$ & $3 m$ \\
\hline
\end{tabular}

Scheme 2. Synthesis of piano-stool vinyl-aminoalkylidene iron complexes from dinuclear precursors; $\mathrm{Xyl}=2,6$ $\mathrm{C}_{6} \mathrm{H}_{3} \mathrm{Me}_{2}, \mathrm{Xyl}-\mathrm{Cl}=2,6-\mathrm{C}_{6} \mathrm{H}_{3} \mathrm{MeCl}$.

Compounds 3a-m were purified by alumina chromatography, and fully characterized by analytical and spectroscopic methods. Furthermore, the molecular structures of $\mathbf{3 b}, \mathbf{3 f}, \mathbf{3 g}$ and $\mathbf{3 h}$ were determined by single-crystal X-ray diffraction (Figure 1, Table 1). 


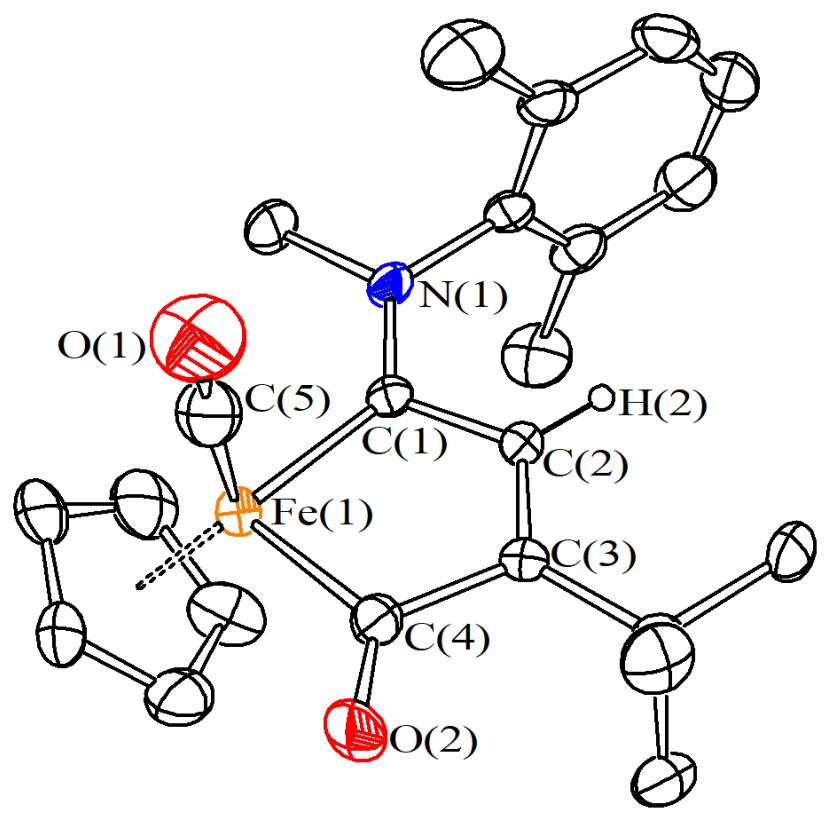

3b

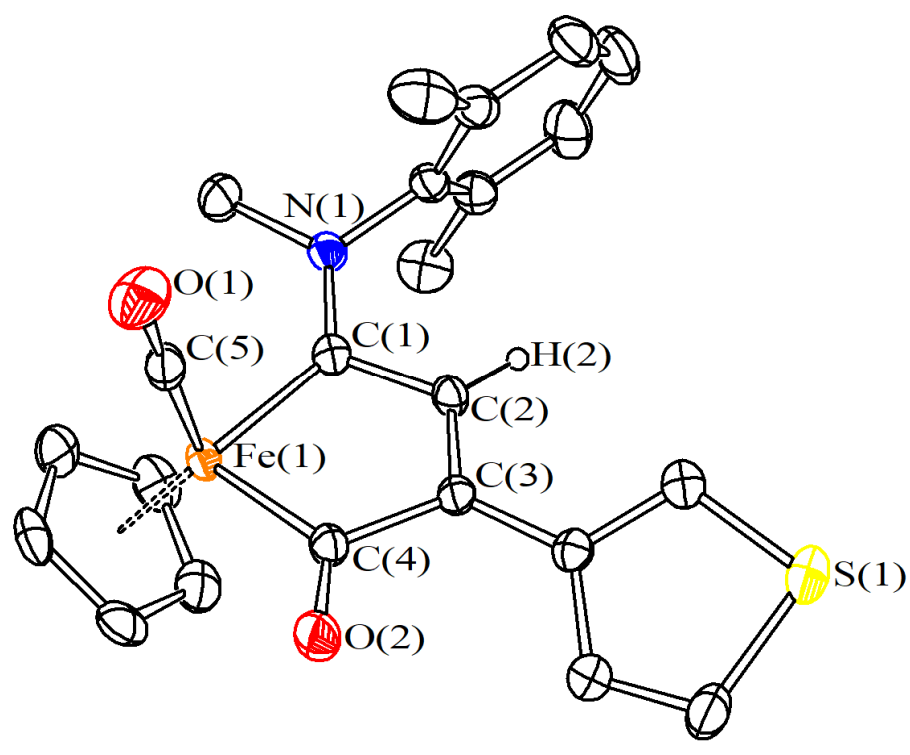

3f

8 

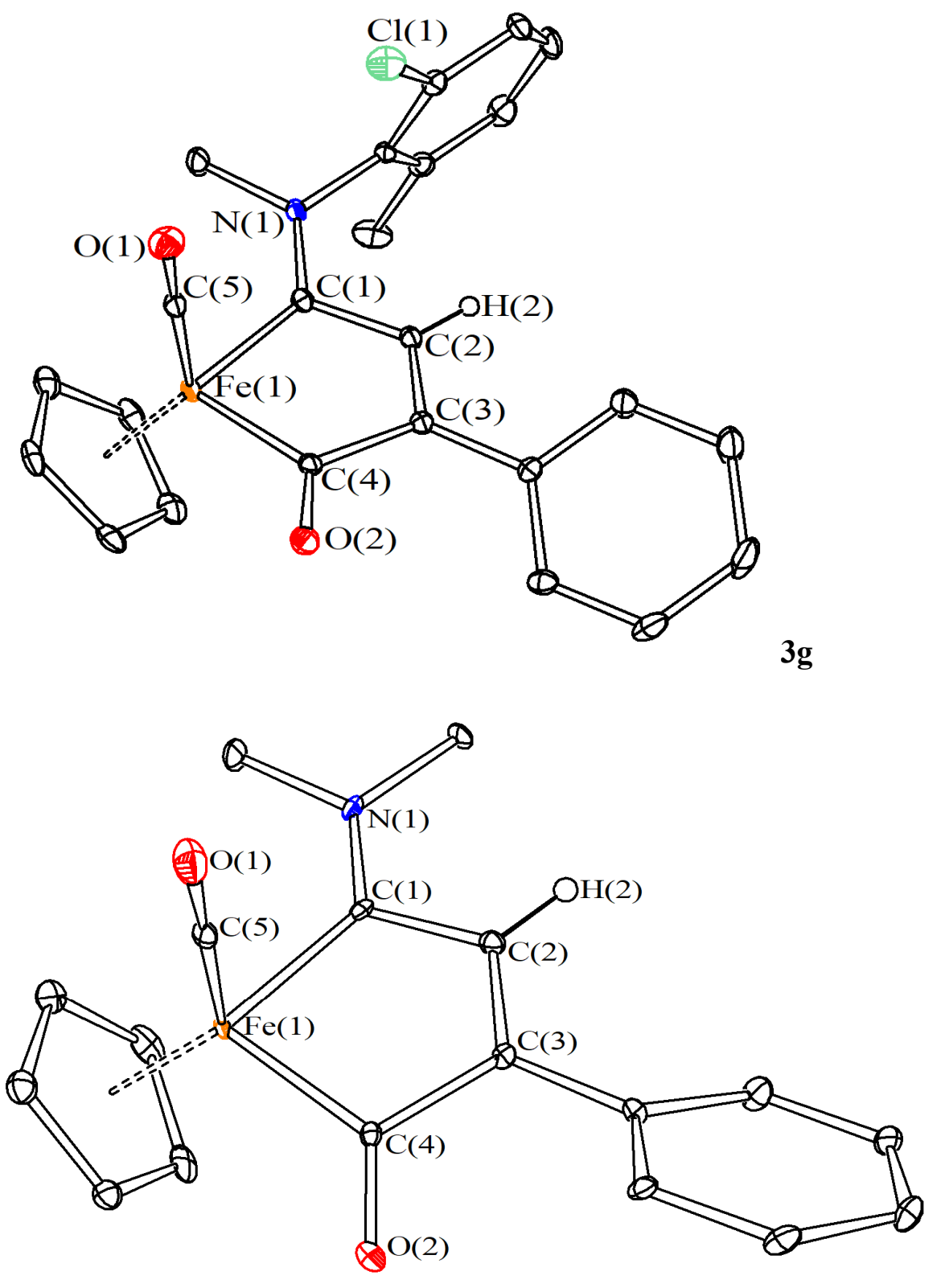

$3 h$

Figure 1. Molecular structures of $\left[\mathrm{FeCp}(\mathrm{CO})\left\{\eta^{2}-\mathrm{C}^{1} \mathrm{Me}(\mathrm{Xyl}) \mathrm{C}^{2} \mathrm{HC}^{3}\left({ }^{\mathrm{t}} \mathrm{Bu}\right) \mathrm{C}(=\mathrm{O})\right\}\right], \quad \mathbf{3 b}, \quad\left[\mathrm{FeCp}(\mathrm{CO})\left\{\eta^{2}-\right.\right.$ $\mathrm{C}^{1} \mathrm{Me}(\mathrm{Xyl}) \mathrm{C}^{2} \mathrm{HC}^{3}\left(3-\right.$ tiophenyl)C(=O)\}], 3f, $\quad\left[\mathrm{FeCp}(\mathrm{CO})\left\{\eta^{2}-\mathrm{C}^{1} \mathrm{Me}(\mathrm{Xyl}-\mathrm{Cl}) \mathrm{C}^{2} \mathrm{HC}^{3}(\mathrm{Ph}) \mathrm{C}(=\mathrm{O})\right\}\right], \quad \mathbf{3 g}, \quad\left[\mathrm{FeCp}(\mathrm{CO})\left\{\eta^{2}-\right.\right.$ $\left.\left.\mathrm{C}^{1} \mathrm{Me}_{2} \mathrm{C}^{2} \mathrm{HC}^{3}(\mathrm{Ph}) \mathrm{C}(=\mathrm{O})\right\}\right]$, 3h. Displacement ellipsoids are at the $30 \%$ probability level. H-atoms, except $\mathrm{H}(4)$, have been omitted for clarity.

Table 1. Selected bond distances $(\AA)$ and angles $\left({ }^{\circ}\right)$ for $\mathbf{3 b}, \mathbf{3 f}, \mathbf{3 g}, \mathbf{3 h}$ and $[4] \mathrm{CF}_{3} \mathrm{SO}_{3}$.

\begin{tabular}{|c|c|c|c|c|c|}
\hline & $\mathbf{3 b}$ & $\mathbf{3 f}$ & $\mathbf{3 g}$ & $\mathbf{3 h}$ & {$[\mathbf{4}] \mathrm{CF}_{3} \mathrm{SO}_{3}$} \\
\hline $\mathrm{Fe}(1)-\mathrm{C}(5)$ & $1.739(7)$ & $1.739(3)$ & $1.746(2)$ & $1.723(7)$ & $1.740(3)$ \\
\hline $\mathrm{Fe}(1)-\mathrm{C}(4)$ & $1.937(5)$ & $1.939(3)$ & $1.936(2)$ & $1.938(6)$ & $1.928(3)$ \\
\hline
\end{tabular}




\begin{tabular}{|c|c|c|c|c|c|}
\hline $\mathrm{Fe}(1)-\mathrm{C}(1)$ & $1.912(4)$ & $1.913(3)$ & $1.905(2)$ & $1.946(6)$ & $1.920(3)$ \\
\hline $\mathrm{C}(5)-\mathrm{O}(1)$ & $1.166(7)$ & $1.148(3)$ & $1.154(3)$ & $1.156(9)$ & $1.142(3)$ \\
\hline $\mathrm{C}(4)-\mathrm{O}(2)$ & $1.208(5)$ & $1.218(3)$ & $1.218(3)$ & $1.216(8)$ & $1.217(3)$ \\
\hline $\mathrm{C}(1)-\mathrm{N}(1)$ & $1.321(5)$ & $1.318(3)$ & $1.325(3)$ & $1.307(8)$ & $1.323(3)$ \\
\hline $\mathrm{C}(3)-\mathrm{C}(4)$ & $1.521(6)$ & $1.525(3)$ & $1.529(3)$ & $1.519(8)$ & $1.524(4)$ \\
\hline $\mathrm{C}(2)-\mathrm{C}(3)$ & $1.326(6)$ & $1.336(4)$ & $1.336(3)$ & $1.329(9)$ & $1.330(4)$ \\
\hline $\mathrm{C}(1)-\mathrm{C}(2)$ & $1.458(6)$ & $1.458(4)$ & $1.463(3)$ & $1.468(9)$ & $1.490(3)$ \\
\hline $\mathrm{Fe}(1)-\mathrm{C}(5)-\mathrm{O}(1)$ & $175.1(6)$ & $178.1(3)$ & $178.2(2)$ & $178.6(6)$ & $178.5(3)$ \\
\hline $\mathrm{Fe}(1)-\mathrm{C}(4)-\mathrm{C}(3)$ & $113.1(3)$ & $112.79(18)$ & $112.97(15)$ & $112.9(4)$ & $112.19(18)$ \\
\hline $\mathrm{C}(4)-\mathrm{C}(3)-\mathrm{C}(2)$ & $112.1(4)$ & $112.3(2)$ & $112.2(2)$ & $113.0(6)$ & $114.3(2)$ \\
\hline $\mathrm{C}(3)-\mathrm{C}(2)-\mathrm{C}(1)$ & $117.0(4)$ & $116.1(2)$ & $115.6(2)$ & $116.0(6)$ & $114.8(2)$ \\
\hline $\mathrm{C}(2)-\mathrm{C}(1)-\mathrm{Fe}(1)$ & $114.0(3)$ & $114.32(18)$ & $114.80(16)$ & $113.5(4)$ & $113.61(18)$ \\
\hline $\mathrm{C}(1)-\mathrm{Fe}(1)-\mathrm{C}(5)$ & $88.2(2)$ & $89.17(11)$ & $83.12(9)$ & $82.9(2)$ & $89.61(12)$ \\
\hline
\end{tabular}

Complexes 3a-m are based on a five-membered metallacycle, consisting of fused vinylaminoalkylidene and acyl units, which is unique in organometallic chemistry. The formation of this structure appears the consequence of the incorporation of the $\mathrm{C}_{3}$ vinyliminium chain, pre-generated on the diiron skeleton from isocyanide/alkyne combination, on the monoiron derivative. Ring closure is ensured by carbon-carbon bond coupling between the $\mathrm{C}^{3}$ carbon and one carbonyl ligand originally present in $\mathbf{2 a - \mathbf { m }}$, forming the acyl group. In analogy with former findings (see above), we propose that

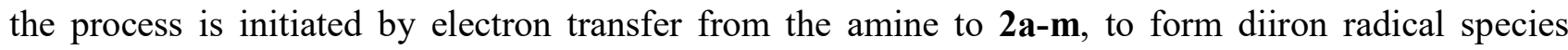
undergoing intramolecular rearrangement until the loss of the $\{\mathrm{FeCp}\}$ unit. ${ }^{[22]}$ Accordingly, when the reaction of $[\mathbf{2 a}] \mathrm{CF}_{3} \mathrm{SO}_{3}$ with pyrrolidine was carried out in air, the IR spectrum of the mixture after 36 hours evidenced only limited conversion of $[\mathbf{2 a}]^{+}$to $\mathbf{3 a}$, suggesting some $\mathrm{O}_{2}$ interference.

It has to be noted that the previously reported generation of vinyl-aminoalkylidene ligands on monoiron compounds suffers from important limitations (see Introduction), including complicated synthetic protocols and restrictions on the nature of the vinyl substituents. ${ }^{[25]}$

The five-membered ring in $\mathbf{3 b} \mathbf{b}, \mathbf{f}, \mathbf{g}, \mathbf{h}$ is almost perfectly planar mean deviations from the $\mathrm{Fe}(1) \mathrm{C}(2)$ $\mathrm{C}(3) \mathrm{C}(4) \mathrm{C}(5)$ least square planes $0.0170,0.0565,0.0549$ and $0.0606 \AA$ for $\mathbf{3 b}, \mathbf{3 f}, \mathbf{3 g}$ and $\mathbf{3 h}$, 
respectively]. The Fe-carbene and $\mathrm{N}$-carbene distances in $\mathbf{3 b}, \mathbf{3 f}, \mathbf{3 g}$ and $\mathbf{3 h}$ [e.g. for $\mathbf{3 b}, \mathrm{Fe}(1)-\mathrm{C}(5)=$ 1.912(4) $\AA$ and $\mathrm{C}(5)-\mathrm{N}(1)=1.321(5) \AA]$ resemble the values reported for other Fe(II)-aminoalkylidene complexes, including 3a and 3o. ${ }^{[20,22]}$ The $C^{2}-C^{3}$ and $C^{1}-C^{2}$ distances [e.g. in 3b: $C(3)-C(4)=1.326(6)$ $\AA, \mathrm{C}(5)-\mathrm{C}(4)=1.458(6) \AA]$ are in agreement with typical $\mathrm{Csp}^{2}=\mathrm{Csp}^{2}$ and $\mathrm{Csp}^{2}-\mathrm{Csp}^{2}$ bond lengths, respectively. ${ }^{[26]}$ In $\mathbf{3 b}, \mathbf{3 f}$ and $\mathbf{3 g}$, the two different N-substituents are oriented in an E-configuration. In the IR spectra of 3a-m (in $\mathrm{CH}_{2} \mathrm{Cl}_{2}$ ), the carbonyl ligand and the acyl group are clearly recognized by two distinct absorptions falling in within the ranges $1909-1922 \mathrm{~cm}^{-1}$ and $1602-1619 \mathrm{~cm}^{-1}$, respectively. The NMR spectra of 3a-f and 3h-l contain a single set of resonances which, in the cases of 3a-f, reasonably correspond to the E configuration of the aminoalkylidene group, consistent with the X-ray structures (see above). Compounds 3a-m contain one stereogenic iron centre, and in principle they exist as a racemic mixture of enantiomers. Instead, two isomers, in nearly 1:1 ratio, were observed for $\mathbf{3 g}$ and $\mathbf{3 m}$. In $\mathbf{3 g}$, probably corresponding to the two possible orientations adopted by the methyl and chlorine arene substituents. Instead, $\mathrm{E}$ and $\mathrm{Z}$ isomers are expected in the case of $\mathbf{3} \mathbf{m}$, due to the comparable steric demands of the methyl and benzyl groups, which bind the nitrogen atom. In the ${ }^{1} \mathrm{H}$ NMR spectra, the vinyl $\mathrm{C}^{2}-\mathrm{H}$ protons are observed in the range $6.45-8.05 \mathrm{ppm}$, while the two Nbound methyl groups give rise to two signals (e.g. at 3.77 and $3.57 \mathrm{ppm}$ in $\mathbf{3 h}$ ), due to the partial double bond nature of $\mathrm{C}^{1}-\mathrm{N}$. Salient ${ }^{13} \mathrm{C}$ NMR features are supplied by the resonances of the metallacyclic carbons. Hence, $\mathrm{C}^{1}$ and $\mathrm{C}^{2}$ are observed in the ranges 256.6 - 268.3 ppm and 146.2 $150.5 \mathrm{ppm}$, in accordance with their amino-alkylidene ${ }^{[21,27]}$ and vinyl character, respectively. The $\mathrm{C}^{3}$ carbon atoms are observed in the range $162.1-183.6 \mathrm{ppm}$, being significantly affected by the nature of R'.

At variance with the general observation that late metal-acyl groups are susceptible to alkylation to afford alkoxy-alkylidene derivatives, ${ }^{[17 a, 28]} \mathbf{3 h}$ is unreactive towards methylating agents (MeI, $\mathrm{CF}_{3} \mathrm{SO}_{3} \mathrm{Me}$ ). Otherwise, the reaction of $\mathbf{3 e}$ with methyl triflate was straightforward and led to selective 
methylation of the pyridyl nitrogen, affording the pyridinium salt $[4] \mathrm{CF}_{3} \mathrm{SO}_{3}$ (Scheme 3). The latter was isolated in $80 \%$ yield after chromatographic purification, and characterized by X-ray diffraction and IR and NMR spectroscopy.

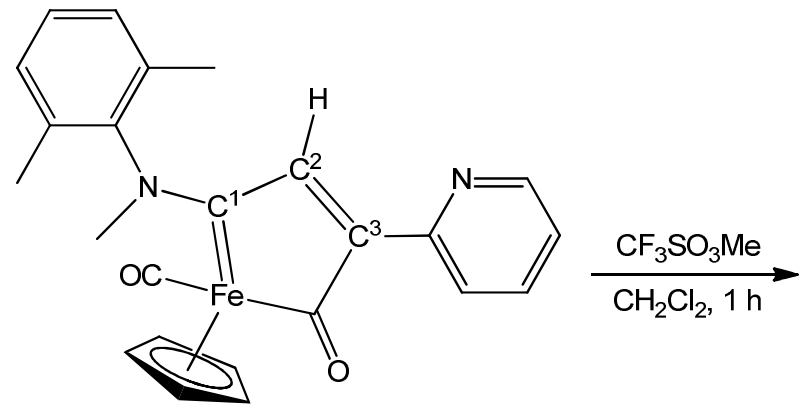

3e

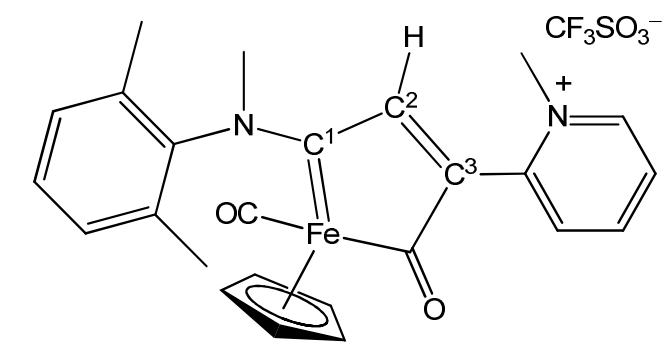

4

Scheme 3. Selective methylation of a pyridyl function in the piano-stool vinyl-aminoalkylidene iron complex $\mathbf{3 e}$.

A view of the structure of the cation is shown in Figure 2, with relevant bonding parameters listed in Table 1 . The most salient aspect of the X-ray structure of $[4]^{+}$is the orientation adopted by the Nsubstituents ( $\mathrm{Z}$ configuration), which is opposite to that observed in the solid state for $\mathbf{3 b} \mathbf{b}, \mathbf{f}, \mathbf{g}$ ( $\mathrm{E}$ configuration). On the other hand, the main bond lengths and angles in $[4]^{+}$do not significantly differ from those in $\mathbf{3 b}, \mathbf{f}, \mathbf{g}, \mathbf{h}$. 


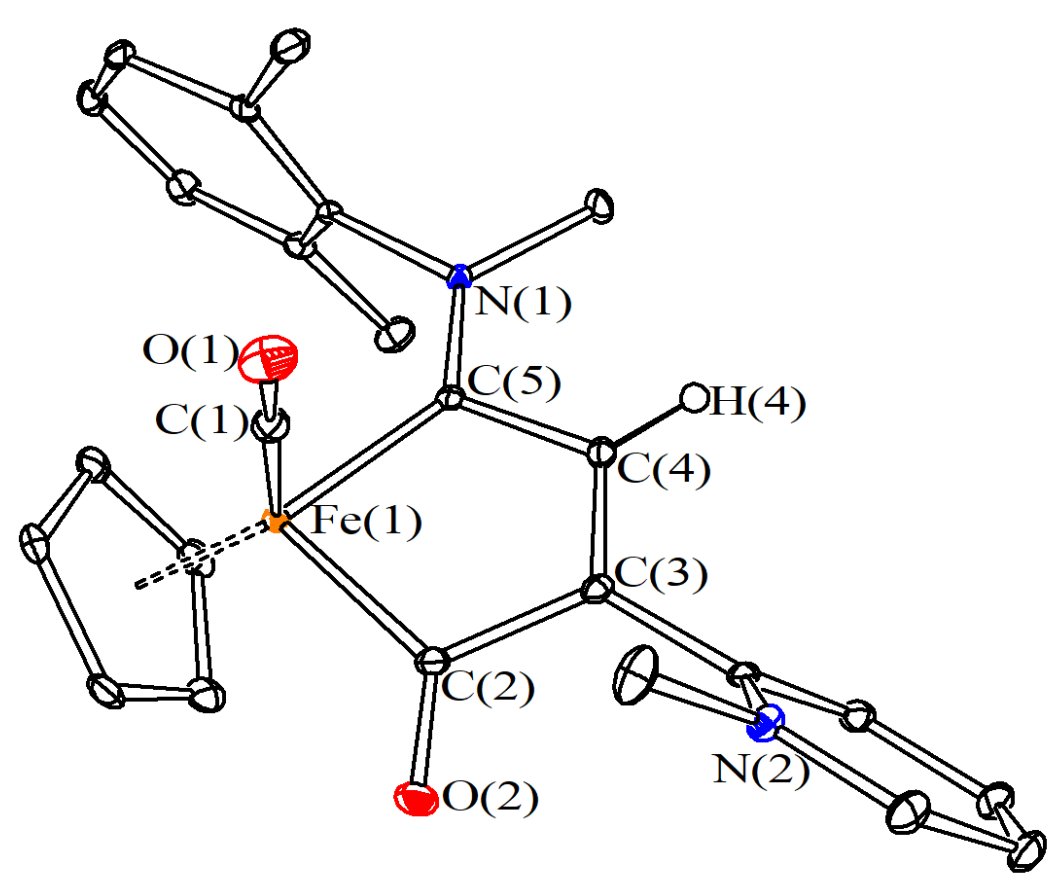

Figure 2. Structure of the cation of $\left[\mathrm{FeCp}(\mathrm{CO})\left\{\mathrm{C}^{1} \mathrm{NMe}(\mathrm{Xyl}) \mathrm{C}^{2} \mathrm{HC}^{3}(2\right.\right.$-methyl-pyridinium $\left.\left.) \mathrm{C}(=\mathrm{O})\right\}\right] \mathrm{CF}_{3} \mathrm{SO}_{3}$, [4] $\mathrm{CF}_{3} \mathrm{SO}_{3}$. Displacement ellipsoids are at the $30 \%$ probability level. $\mathrm{H}$-atoms, except $\mathrm{H}(4)$, have been omitted for clarity.

Methylation of the pyridyl moiety in $\mathbf{3 e}$ produces a notable variation of the electron density at the iron atom, as evidenced by the IR stretching frequency of the carbonyl ligand which moves from 1919 in $3 \mathbf{e}$ to $1939 \mathrm{~cm}^{-1}$ in $\left[4^{+}\right]$. Conversely, the conversion of $\mathbf{3 e}$ to $[4] \mathrm{CF}_{3} \mathrm{SO}_{3}$ does not give rise to major changes in the NMR spectrum. In particular, the $C^{1}, C^{2}$ and $C^{3}$ resonance values of $\left[4^{+}\right](262.8,149.9$ and $167.0 \mathrm{ppm}$, respectively, $\mathrm{dmso}^{6} \mathrm{~d}^{6}$ solution) are close to the corresponding ones observed for $\mathbf{3 e}$ (263.2, 152.5 and $161.4 \mathrm{ppm}$, respectively, $\mathrm{CD}_{2} \mathrm{Cl}_{2}$ solution).

Relevant to the biological studies, the stability of compounds $\mathbf{3 a - m}$ and $[4] \mathrm{CF}_{3} \mathrm{SO}_{3}$ was monitored in $\mathrm{D}_{2} \mathrm{O} / \mathrm{dmso} \mathrm{d}_{6}$ solutions by ${ }^{1} \mathrm{H}$ NMR spectroscopy, after storing the solutions at $37{ }^{\circ} \mathrm{C}$ for 72 hours: all the compounds are substantially stable, since only traces $(<10 \%)$ of additional species were NMR detected at the end of treatment. Moreover, the compounds showed robustness even in dmso/cell culture medium mixture (RPMI-1640), and were cleanly recovered after 72 hours at $37{ }^{\circ} \mathrm{C}$ following dichloromethane extraction (see Experimental for details). 


\section{Electrochemistry.}

The electrochemical behaviour of selected monoiron compounds was investigated in acetonitrile solution (and also in tetrahydrofuran in some cases) by cyclic voltammetry at ambient temperature. The results are summarized in Table 2; as a representative example, the voltammogram of $\mathbf{3 d}$ in $\mathrm{MeCN}$ is reported in Figure 3, while all cyclic voltammetry profiles are supplied as Supporting Information (Figures S44-S56).

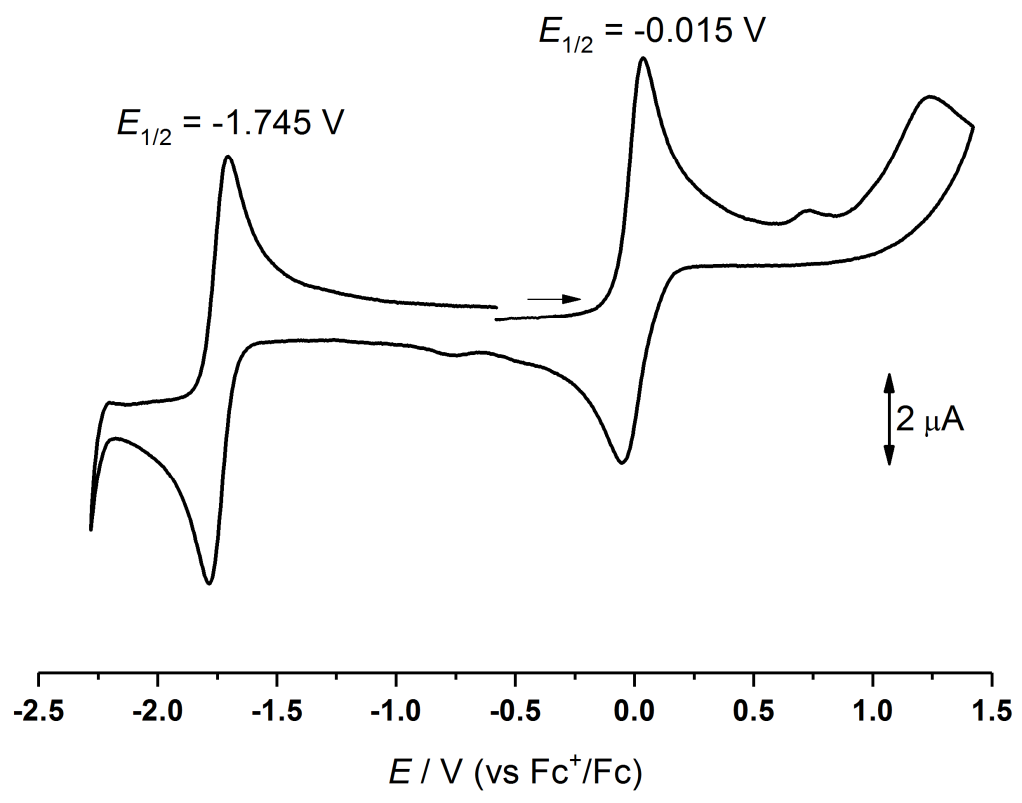

Figure 3. Cyclic voltammogram of $\mathbf{3 d}$ recorded in $\mathrm{MeCN}$ at a scan rate of $100 \mathrm{mV} / \mathrm{s}$ with $\mathrm{NBu}_{4} \mathrm{PF}_{6}(0.1 \mathrm{M})$ as the supporting electrolyte; potentials vs $\mathrm{Fc}^{+} / \mathrm{Fc}$.

In general, two main electron transfers were observed with respect to the ferrocene/ferrocenium redox couple. More precisely, the compounds (in acetonitrile) exhibit one oxidation (ascribable to the $\mathrm{Fe}^{\mathrm{II}} / \mathrm{Fe}^{\mathrm{III}}$ couple) and one reduction in the potential ranges $-0.435 \mathrm{~V}$ to $0.015 \mathrm{~V}$ and $-2.240 \mathrm{~V}$ to -1.675 $\mathrm{V}$, respectively. Both anodic and cathodic processes are generally reversible in the time scale of the experiment, as suggested by the peak-to-peak separation for each couple being lower than $100 \mathrm{mV}$ 
(The theoretical value is $59 \mathrm{mV}$ at room temperature for a reversible one-electron event with fast electron-transfer kinetics). ${ }^{[29]}$ The only exception is given by complex $\mathbf{3 j}$, showing a two-step electrochemical oxidation at $-0.20 \mathrm{~V}$ and an irreversible reduction at $-2.24 \mathrm{~V}$. This peculiar behaviour may be related to the combination of electron donor R and R' substituents (i.e., methyl and tert-butyl), probably affecting the stability of the formally reduced species $[\mathbf{3 j}]^{-}$, this latter undergoing chemical transformation after electron transfer.

Table 2. Overview of the first oxidation and reduction potentials $\left(\mathrm{V} \mathrm{vs} . \mathrm{Fc}^{+} / \mathrm{Fc}\right)$ at a scan rate of $100 \mathrm{mV} / \mathrm{s}$ determined by cyclic voltammetry for a selection of monoiron complexes. The peak-to peak separation $\left(\Delta \mathrm{E}_{\mathrm{p}}\right)$ is defined by the difference between the two peak potentials for a given redox couple.

\begin{tabular}{cccccc}
\hline Compound & Solvent & Oxidation & $\Delta \mathrm{E}_{\mathrm{p}}(\mathbf{o x})$ & Reduction & $\Delta \mathrm{E}_{\mathrm{p}}(\mathbf{r e d})$ \\
\hline $\mathbf{3 a}$ & $\mathrm{MeCN}$ & -0.160 & 83 & -1.940 & 70 \\
\hline $\mathbf{3 a}$ & $\mathrm{THF}$ & -0.135 & - & -2.000 & - \\
\hline $\mathbf{3} \mathbf{c}$ & $\mathrm{MeCN}$ & -0.105 & 100 & -2.135 & 80 \\
\hline $\mathbf{3 d}$ & $\mathrm{MeCN}$ & -0.029 & 65 & -1.860 & 80 \\
\hline $\mathbf{3 e}$ & $\mathrm{MeCN}$ & -0.015 & 85 & -1.745 & 80 \\
\hline $\mathbf{3 f}$ & $\mathrm{MeCN}$ & -0.145 & 96 & -1.935 & 95 \\
\hline $\mathbf{3 h}$ & $\mathrm{MeCN}$ & -0.370 & 80 & -2.090 & 83 \\
\hline $\mathbf{3 i}$ & $\mathrm{MeCN}$ & -0.435 & 91 & -2.020 & 80 \\
\hline $\mathbf{3 i}$ & $\mathrm{THF}$ & -0.155 & - & -1.830 & - \\
\hline $\mathbf{3 j}$ & $\mathrm{MeCN}$ & $-0.200(2 \mathrm{steps})$ & 100 & -2.240 (irr) & - \\
\hline $\mathbf{3 l}$ & $\mathrm{MeCN}$ & -0.080 & 81 & -1.795 & 82 \\
\hline $\mathbf{3 n} \mathbf{n}^{22}$ & $\mathrm{MeCN}$ & -0.180 & 81 & -2.070 & 90 \\
\hline & & & & & \\
\hline
\end{tabular}

\section{Cytotoxicity studies.}


The cytotoxicity of the new compounds $\mathbf{3 b}-\mathbf{m}$ and $[4] \mathrm{SO}_{3} \mathrm{CF}_{3}$ was assessed against cisplatin sensitive and cisplatin resistant human ovarian carcinoma (A2780 and A2780cisR) cell lines and the nontumorigenic human embryonic kidney (HEK-293) cell line. Cisplatin and $\left[\mathrm{RuCl}_{2}\left(\eta^{6}-\mathrm{p}-\mathrm{cymene}\right)(\kappa P-\right.$ pta)] (RAPTA-C $)^{[30]}$ were evaluated as positive and negative controls, respectively. The obtained $\mathrm{IC}_{50}$ values are presented in Table 3, where they are compared to those previously determined for $\mathbf{3 a}$ and $\mathbf{3 n}$. The compounds display a range of cytotoxicities, with $\mathbf{3 b}$ displaying the lowest $\mathrm{IC}_{50}$ values in the two cancer cell lines $(6.7 \pm 1.2 \mu \mathrm{M}$ in $\mathrm{A} 2780$ cells and $11 \pm 1 \mu \mathrm{M}$ in A2780cisR cells) together with reasonable degree of cancer cell selectivity $(41 \pm 10 \mu \mathrm{M}$ in HEK-293 cells). Compound $\mathbf{3 f}$ contains a thiophenyl group, which is known to impart various pharmacological properties to organic scaffolds, including anticancer activity. ${ }^{[31]}$ In keeping with 3a, the presence of a phenyl ring attached to the metallacycle appears to favourably affect the selectivity (3g and $\mathbf{3 h}$ ), with the compounds being essentially inactive in the HEK-293 cell line.

Previous studies on 3a $\left(\mathrm{R}^{\prime}=\mathrm{Ph}\right)$ indicated that the antiproliferative activity is likely related to a combination of the accessible oxidation potential, triggering the production of ROS, and the compact, hydrophobic structure, allowing DNA binding. ${ }^{[20]}$ Accordingly, the lack of cytotoxicity detected for 3n $\left(\mathrm{R}^{\prime}=\mathrm{CH}_{2} \mathrm{OH}\right)$ was ascribed to a less favourable DNA binding, obstructed by the hydrophilic hydroxyl unit. ${ }^{[20]}$ Based on the electrochemical results, all the neutral compounds (3) might be prone to oxidation inside the cells, and thus represent further examples of anticancer metal-based compounds that are potentially activated via an oxidation mechanism, ${ }^{[3 \mathrm{a}-\mathrm{b}, 32]}$ as opposed to the more commonly encountered activation by reduction mechanism. ${ }^{[33]}$ It should be noted, however, that there is not a clear correlation between the oxidation and reduction potentials of 3a-n (Table 2) and their cytotoxicities (Table 3). Such disparity is not unexpected as other factors which also impact on cytotoxicity, such as cellular uptake, will vary across the series of compounds. The inactivity of $[4] \mathrm{CF}_{3} \mathrm{SO}_{3}$ may be related to its ionic nature, disfavouring both cellular uptake and oxidation. It should also be noted that 3a-n tend to be less cytotoxic than their bimetallic precursors, $\mathbf{2 a - n},{ }^{[20]}$ and that the trends in cytotoxicities between 
the two groups of complexes deviate substantially, despite the bimetallic complexes potentially undergoing fragmentation in vitro to form mononuclear species. In general, bimetallic complexes are often more cytotoxic than mononuclear complexes, irrespective of whether they remain intact or fragment following cellular uptake, ${ }^{[34]}$ although many highly cytotoxic mononuclear iron-based organometallic complexes are known. ${ }^{[3 b, 35]}$

Table 3. $\mathrm{IC}_{50}$ values $(\mu \mathrm{M})$ determined for compounds 3a-n, $[4] \mathrm{CF}_{3} \mathrm{SO}_{3}$ and cisplatin on human ovarian carcinoma (A2780), human ovarian carcinoma cisplatin resistant (A2780CisR) and human embryonic kidney (HEK-293) cell lines after $72 \mathrm{~h}$ exposure. Values are given as the mean \pm SD.

\begin{tabular}{cccc}
\hline Compound & A2780 & A2780cisR & HEK-293 \\
\hline $\mathbf{3 a}^{20}$ & $16 \pm 2$ & $26 \pm 3$ & $>200$ \\
$\mathbf{3 b}$ & $6.7 \pm 1.2$ & $11 \pm 1$ & $41 \pm 10$ \\
$\mathbf{3 c}$ & $11 \pm 2$ & $31 \pm 3$ & $7.0 \pm 1.3$ \\
$\mathbf{3 d}$ & $46 \pm 3$ & $46 \pm 3$ & $34 \pm 3$ \\
$\mathbf{3 e}$ & $31 \pm 1$ & $31 \pm 2$ & $69 \pm 6$ \\
$\mathbf{3 f}$ & $8 \pm 2$ & $>200$ & $37 \pm 2$ \\
$\mathbf{3 g}$ & $20 \pm 2$ & $17.7 \pm 1.1$ & $>200$ \\
$\mathbf{3 h}$ & $44 \pm 3$ & $30 \pm 2$ & $>200$ \\
$\mathbf{3 i}$ & $31 \pm 4$ & $19 \pm 2$ & $43 \pm 7$ \\
$\mathbf{3 j}$ & $37 \pm 3$ & $22 \pm 3$ & $44 \pm 10$ \\
$\mathbf{3 k}$ & $21 \pm 2$ & $34 \pm 2$ & $24 \pm 3$ \\
$\mathbf{3 l}$ & $>200$ & $>200$ & $170 \pm 15$ \\
$\mathbf{3 m}$ & $13.4 \pm 0.8$ & $9.4 \pm 1.1$ & $38 \pm 3$ \\
$\mathbf{3 n}^{20}$ & $180 \pm 1$ & $>200$ & $>200$ \\
{$[\mathbf{4}] \mathrm{CF}_{3} \mathrm{SO}_{3}$} & $>200$ & $>200$ & $>200$ \\
cisplatin $^{20}$ & $2.3 \pm 0.6$ & $31 \pm 3$ & $8.4 \pm 0.9$ \\
\hline
\end{tabular}

\section{Conclusions}

Bimetallic complexes are ideal for constructing functionalized organic and organometallic architectures and the readily available dimer $\mathrm{Fe}_{2} \mathrm{Cp}_{2}(\mathrm{CO})_{4}$ represents a versatile starting material. In particular, bridging vinyliminium ligands with unusual coordination fashion can be fabricated on a diiron frame 
by stepwise assembly of one isocyanide and one alkyne. Herein, we have described the aminepromoted selective fragmentation of easily available diiron vinyliminium complexes into piano stool complexes featuring a unique structural motif, which arises from the adaptation of the intact vinyliminium moiety to the monoiron species. Structural variation is guaranteed by the general character of the reaction, and the availability of a large number of alkynes. In particular, the introduction of a pyridyl group allows subsequent derivatization via alkylation. The series of piano stool iron compounds exhibits a variable cytotoxic activity, and a selectivity against cancer cell lines seems favoured by the presence on the vinyl moiety of a phenyl substituent rather than other groups.

\section{Experimental}

Materials and methods. All manipulations were carried out under $\mathrm{N}_{2}$ atmosphere using standard Schlenk techniques. The reaction vessels were oven dried at $120^{\circ} \mathrm{C}$ prior to use, evacuated $\left(10^{-2}\right.$ $\mathrm{mmHg}$ ) and then filled with $\mathrm{N}_{2}$. Organic reactants (TCI Europe or Sigma Aldrich) and $\mathrm{Fe}_{2} \mathrm{Cp}_{2}(\mathrm{CO})_{4}$ (Strem) were commercial products of the highest purity available. Compounds $\left[\mathrm{Fe}_{2} \mathrm{Cp}_{2}(\mathrm{CO})_{2}(\mu-\mathrm{CO})\{\mu-\right.$ $\mathrm{CNMe}(\mathrm{R})\}] \mathrm{CF}_{3} \mathrm{SO}_{3}\left(\mathrm{R}=\mathrm{Xyl}=2,6-\mathrm{C}_{6} \mathrm{H}_{3} \mathrm{Me}_{2},[\mathbf{1} \mathbf{a}] \mathrm{CF}_{3} \mathrm{SO}_{3} ; \mathrm{R}=\mathrm{Xyl}-\mathrm{Cl}=2,6-\mathrm{C}_{6} \mathrm{H}_{3} \mathrm{MeCl},[\mathbf{1} \mathbf{b}] \mathrm{CF}_{3} \mathrm{SO}_{3}\right.$; $\left.\mathrm{R}=\mathrm{Me},[\mathbf{1} \mathbf{c}] \mathrm{CF}_{3} \mathrm{SO}_{3} ; \mathrm{R}=\mathrm{CH}_{2} \mathrm{Ph},[\mathbf{1 d}] \mathrm{CF}_{3} \mathrm{SO}_{3}\right),{ }^{[18]}[\mathbf{2} \mathbf{a}, \mathbf{c}, \mathbf{e}, \mathbf{f}, \mathbf{h}, \mathbf{k}] \mathrm{CF}_{3} \mathrm{SO}_{3},{ }^{[20]}$ and $\mathbf{3 n}{ }^{[22]}$ were prepared according to published procedures. Solvents were distilled before use under $\mathrm{N}_{2}$ from appropriate drying agents. Chromatography was carried out under $\mathrm{N}_{2}$ on deactivated alumina columns (Sigma Aldrich, 4\% $w / w$ water). Infrared spectra of solid samples were recorded on a Perkin Elmer Spectrum One FT-IR spectrometer, equipped with a UATR sampling accessory (4000-400 $\mathrm{cm}^{-1}$ range). Infrared spectra of solutions were recorded on a Perkin Elmer Spectrum 100 FT-IR spectrometer with a $\mathrm{CaF}_{2}$ liquid transmission cell (2300-1500 $\mathrm{cm}^{-1}$ range). NMR spectra were recorded at $298 \mathrm{~K}$ on a Bruker Avance II DRX400 instrument equipped with a BBFO broadband probe. Chemical shifts (expressed in parts per million) are referenced to the residual solvent peaks ${ }^{[36]}\left({ }^{1} \mathrm{H},{ }^{13} \mathrm{C}\right)$ or to external standard $\left({ }^{19} \mathrm{~F}, \mathrm{CFCl}_{3}\right)$. 
NMR spectra were assigned with the assistance of ${ }^{1} \mathrm{H}^{13} \mathrm{C}(g s-\mathrm{HSQC}$ and $g s-\mathrm{HMBC})$ correlation experiments. ${ }^{[37]}$ NMR signals due to a second isomeric form (where relevant) are italicized. Carbon, hydrogen and nitrogen analyses were performed on a Vario MICRO cube instrument (Elementar).

\section{1) Synthesis and characterization of $[2] \mathrm{CF}_{3} \mathrm{SO}_{3}$.}

General procedure. The appropriate precursor $[1] \mathrm{CF}_{3} \mathrm{SO}_{3}$ (ca. $0.5 \mathrm{mmol}$ ) was dissolved into acetonitrile $(10 \mathrm{~mL})$, then $\mathrm{Me}_{3} \mathrm{NO}$ (1.3 eq.) was added. The resulting mixture was stirred for $1 \mathrm{~h}$, and progressive darkening of the solution was observed. The conversion of the starting material into the acetonitrile adduct $\left[\mathrm{Fe}_{2} \mathrm{Cp}_{2}(\mathrm{CO})(\mu-\mathrm{CO})(\mathrm{NCMe})\{\mu-\mathrm{CNMe}(\mathrm{R})\}\right] \mathrm{CF}_{3} \mathrm{SO}_{3}$ (Scheme 1) was confirmed by IR spectroscopy. The volatiles were removed under vacuum, and the residue was dissolved in dichloromethane (ca. $20 \mathrm{~mL}$ ). The solution was treated with the appropriate alkyne (ca. 1.3 eq.), and the mixture was stirred at room temperature for $48 \mathrm{~h}$ under a $\mathrm{N}_{2}$ atmosphere. The final mixture was charged on an alumina column. Elution with $\mathrm{CH}_{2} \mathrm{Cl}_{2}$ and $\mathrm{CH}_{2} \mathrm{Cl}_{2} / \mathrm{THF}$ mixtures allowed unreacted alkyne and impurities to be removed, then a fraction corresponding to the desired product was collected using a $\mathrm{CH}_{3} \mathrm{CN} / \mathrm{MeOH}$ mixture $(9: 1 \mathrm{v} / \mathrm{v})$ as eluent. Removal of the solvent under reduced pressure afforded an air stable solid.

$\left[\mathrm{Fe}_{2} \mathrm{Cp}_{2}(\mathrm{CO})(\mu-\mathrm{CO})\left\{\mu-\eta^{1}: \eta^{3}-\mathrm{C}^{3}\left({ }^{\mathrm{B}} \mathrm{Bu}\right) \mathrm{C}^{2} \mathrm{HC}{ }^{1} \mathrm{~N}(\mathrm{Me})(\mathrm{Xyl})\right\}\right] \mathrm{CF}_{3} \mathrm{SO}_{3},[2 \mathrm{~b}] \mathrm{CF}_{3} \mathrm{SO}_{3}$ (Chart 1).

Chart 1. Structure of [2b] . 


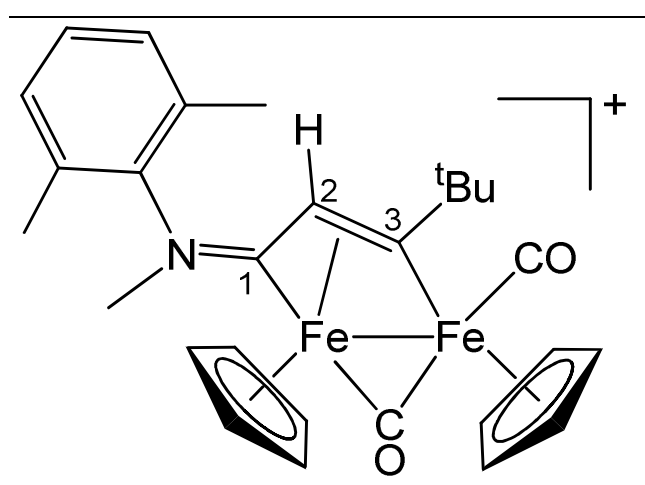

From $[\mathbf{1 a}] \mathrm{CF}_{3} \mathrm{SO}_{3}$ and 3,3-dimethyl-1-butyne. Brown-yellow solid, yield 58\%. Anal. calcd. for $\mathrm{C}_{29} \mathrm{H}_{32} \mathrm{~F}_{3} \mathrm{Fe}_{2} \mathrm{NO}_{5} \mathrm{~S}: \mathrm{C}, 51.58 ; \mathrm{H}, 4.78 ; \mathrm{N}, 2.07$. Found: C, 51.37; H, 4.88; N, 1.98. IR $\left(\mathrm{CH}_{2} \mathrm{Cl}_{2}\right): \tilde{v} / \mathrm{cm}^{-1}=$ 1999vs (CO), 1804s $(\mu-\mathrm{CO}), 1635 \mathrm{~m}\left(\mathrm{C}^{2} \mathrm{C}^{1} \mathrm{~N}\right) .{ }^{1} \mathrm{H}$ NMR (acetone-d $\left.)_{6}\right): \delta / \mathrm{ppm}=7.25-7.03(\mathrm{~m}, 3 \mathrm{H}$, $\left.\mathrm{C}_{6} \mathrm{H}_{3} \mathrm{Me}_{2}\right) ; 5.75,5.53(\mathrm{~s}, 10 \mathrm{H}, \mathrm{Cp}) ; 4.40(\mathrm{~s}, 3 \mathrm{H}, \mathrm{NMe}) ; 4.29\left(\mathrm{~s}, 1 \mathrm{H}, \mathrm{C}^{2} \mathrm{H}\right) ; 2.33,1.89(\mathrm{~s}, 6 \mathrm{H}$, $\left.\mathrm{C}_{6} \mathrm{H}_{3} M e_{2}\right) ; 1.76\left(\mathrm{~s}, 9 \mathrm{H}, \mathrm{CMe}_{3}\right) .{ }^{13} \mathrm{C}\left\{{ }^{1} \mathrm{H}\right\}$ NMR (acetone-d $\left.\mathrm{d}_{6}\right): \delta / \mathrm{ppm}=256.6(\mu-\mathrm{CO}) ; 231.2\left(\mathrm{C}^{1}\right) ; 225.4$ $\left(\mathrm{C}^{3}\right) ; 211.2$ (CO); 145.4 (ipso- $\left.C_{6} \mathrm{H}_{3} \mathrm{Me}_{2}\right)$; 131.7, 131.6, 129.5, $129.3\left(C_{6} \mathrm{H}_{3} \mathrm{Me}_{2}\right) ; 90.9,87.2(\mathrm{Cp}) ; 49.4$ $\left(C \mathrm{Me}_{3}\right) ; 49.1\left(\mathrm{C}^{2}\right) ; 45.2(\mathrm{NMe}) ; 34.5\left(\mathrm{CMe}_{3}\right) ; 17.2,16.6\left(\mathrm{C}_{6} \mathrm{H}_{3} \mathrm{Me}_{2}\right)$.

\section{$\left[\mathrm{Fe}_{2} \mathrm{Cp}_{2}(\mathrm{CO})(\mu-\mathrm{CO})\left\{\mu-\eta^{1}: \eta^{3}-\mathrm{C}^{3}(3-\mathrm{Py}) \mathrm{C}^{2} \mathrm{HC}^{1} \mathrm{~N}(\mathrm{Me})(\mathrm{Xyl})\right\}\right] \mathrm{CF}_{3} \mathrm{SO}_{3},[2 \mathrm{~d}] \mathrm{CF}_{3} \mathrm{SO}_{3}$ (Chart 2).}

Chart 2. Structure of $[\mathbf{2 d}]^{+}$.

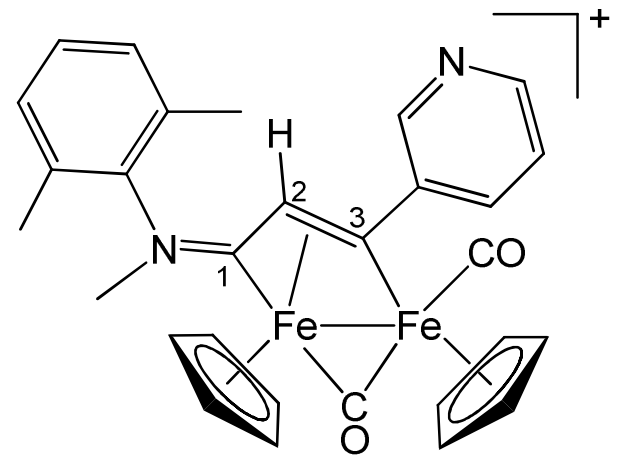

From $[\mathbf{1 a}] \mathrm{CF}_{3} \mathrm{SO}_{3}$ and 3-ethynylpyridine. Brown-green solid, yield 90\%. Anal. calcd. for $\mathrm{C}_{30} \mathrm{H}_{27} \mathrm{~F}_{3} \mathrm{Fe}_{2} \mathrm{~N}_{2} \mathrm{O}_{5} \mathrm{~S}: \mathrm{C}, 51.75 ; \mathrm{H}, 3.91 ; \mathrm{N}, 4.02$. Found: C, 51.61; H, 3.86; N, 4.13. IR $\left(\mathrm{CH}_{2} \mathrm{Cl}_{2}\right): \tilde{v} / \mathrm{cm}^{-1}$ $=2004 \mathrm{vs}(\mathrm{CO}), 1820 \mathrm{~s}(\mu-\mathrm{CO}), 1632 \mathrm{~m}\left(\mathrm{C}^{2} \mathrm{C}^{1} \mathrm{~N}\right) .{ }^{1} \mathrm{H}$ NMR $\left(\right.$ acetone- $\left._{6}\right): \delta / \mathrm{ppm}=8.70,8.11,7.60-7.06$ 
$\left(\mathrm{m}, 7 \mathrm{H}, \mathrm{C}_{5} \mathrm{H}_{4} \mathrm{~N}+\mathrm{C}_{6} \mathrm{H}_{3} \mathrm{Me}_{2}\right) ; 5.72,5.45,5.39,5.11(\mathrm{~s}, 10 \mathrm{H}, \mathrm{Cp}) ; 4.44(\mathrm{~s}, 3 \mathrm{H}, \mathrm{NMe}) ; 3.77(\mathrm{~s}, 1 \mathrm{H}$, $\left.\mathrm{C}^{2} \mathrm{H}\right) ; 2.35,1.89\left(\mathrm{~s}, 6 \mathrm{H}, \mathrm{C}_{6} \mathrm{H}_{3} M e_{2}\right) . \mathrm{E} / \mathrm{Z}$ ratio $=$ ca. $4 .{ }^{13} \mathrm{C}\left\{{ }^{1} \mathrm{H}\right\} \mathrm{NMR}\left(\mathrm{dmso}_{6}\right): \delta / \mathrm{ppm}=232.1\left(\mathrm{C}^{1}\right)$; $210.0(\mathrm{CO}) ; 148.2-123.1\left(\mathrm{C}_{5} \mathrm{H}_{4} \mathrm{~N}+C_{6} \mathrm{H}_{3} \mathrm{Me}_{2}\right) ; 92.4,88.3(\mathrm{Cp}) ; 54.2\left(\mathrm{C}^{2}\right) ; 45.6(\mathrm{NMe}) ; 17.4,16.6$ $\left(\mathrm{C}_{6} \mathrm{H}_{3} M e_{2}\right)$.

$\left[\mathrm{Fe}_{2} \mathrm{Cp}_{2}(\mathrm{CO})(\mu-\mathrm{CO})\left\{\mu-\eta^{1}: \eta^{3}-\mathrm{C}^{3}(\mathrm{Ph}) \mathrm{C}^{2} \mathrm{HC}^{1} \mathrm{~N}(\mathrm{Me})\left(\mathrm{Xyl}^{\mathrm{Cl}}\right)\right\}\right] \mathrm{CF}_{3} \mathrm{SO}_{3},[2 \mathrm{~g}] \mathrm{CF}_{3} \mathrm{SO}_{3}(\mathrm{Chart} 3)$

Chart 3. Structure of $[\mathbf{2 g}]^{+}$.

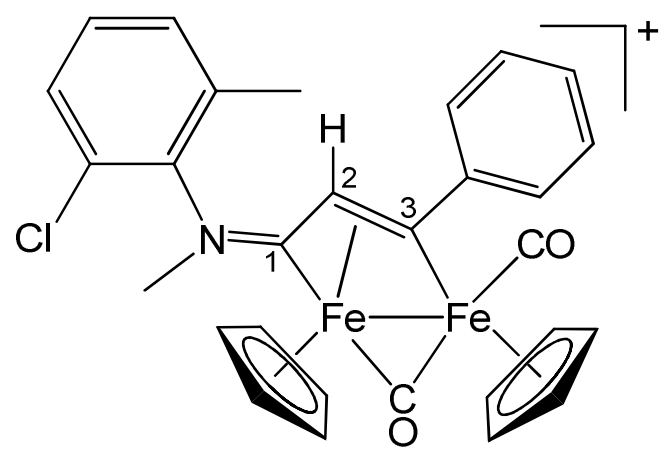

From $[\mathbf{1 b}] \mathrm{CF}_{3} \mathrm{SO}_{3}$ and phenylacetylene. Greenish-brown solid, yield 75\%. Anal. calcd. for $\mathrm{C}_{30} \mathrm{H}_{25} \mathrm{ClF}_{3} \mathrm{Fe}_{2} \mathrm{NO}_{5} \mathrm{~S}: \mathrm{C}, 50.34 ; \mathrm{H}, 3.52 ; \mathrm{N}, 1.96$. Found: C, 50.16; H, 3.61; N, 2.07. IR $\left(\mathrm{CH}_{2} \mathrm{Cl}_{2}\right): \tilde{v} / \mathrm{cm}^{-}$ ${ }^{1}=2005 \mathrm{vs}(\mathrm{CO}), 1822 \mathrm{~s}(\mu-\mathrm{CO}), 1607 \mathrm{~s}\left(\mathrm{C}^{2} \mathrm{C}^{1} \mathrm{~N}\right) .{ }^{1} \mathrm{H}$ NMR $\left(\right.$ acetone- $\left.\mathrm{d}_{6}\right): \delta / \mathrm{ppm}=7.60,7.50,7.37(\mathrm{~m}$, $\left.8 \mathrm{H}, \mathrm{Ph}+\mathrm{C}_{6} \mathrm{H}_{3} \mathrm{ClMe}\right) ; 5.72,5.45,5.41(\mathrm{~s}, 10 \mathrm{H}, \mathrm{Cp}) ; 4.77\left(\mathrm{~s}, 1 \mathrm{H}, \mathrm{C}^{2} \mathrm{H}\right) ; 4.48$ (s, $\left.3 \mathrm{H}, \mathrm{NMe}\right) ; 2.45,2.10$, $1.98\left(\mathrm{~s}, 3 \mathrm{H}, \mathrm{C}_{6} \mathrm{H}_{3} M e\right)$. Isomer ratio $\cong 1 .{ }^{13} \mathrm{C}\left\{{ }^{1} \mathrm{H}\right\}$ NMR (acetone- $\left.\mathrm{d}_{6}\right): \delta / \mathrm{ppm}=253.3,252.5(\mu-\mathrm{CO}) ;$ 235.5, $234.3\left(\mathrm{C}^{1}\right) ; 210.6,210.3,204.9,208.2\left(\mathrm{CO}+\mathrm{C}^{3}\right) ; 156.2\left(\right.$ ipso- $\left.\mathrm{C}_{6} \mathrm{H}_{3}\right) ; 143.0,135.5,131.1-126.9$ $\left(\mathrm{C}_{6} \mathrm{H}_{3}\right) ; 92.8,92.6,88.6,88.4(\mathrm{Cp}) ; 54.6,54.3\left(\mathrm{C}^{2}\right) ; 53.9,45.6(\mathrm{NMe}) ; 18.0,16.9\left(\mathrm{C}_{6} \mathrm{H}_{3} M e\right)$.

$\left[\mathrm{Fe}_{2} \mathrm{Cp}_{2}(\mathrm{CO})(\mu-\mathrm{CO})\left\{\mu-\eta^{1}: \eta^{3}-\mathrm{C}^{3}\left(3,5-\mathrm{C}_{6} \mathrm{H}_{3}\left(\mathrm{CF}_{3}\right)_{2}\right) \mathrm{C}^{2} \mathrm{HC}^{1} \mathrm{NMe}_{2}\right\}\right] \mathrm{CF}_{3} \mathrm{SO}_{3},[2 \mathrm{i}] \mathrm{CF}_{3} \mathrm{SO}_{3}$ (Chart 4).

Chart 4. Structure of $[2 \mathbf{i}]^{+}$. 


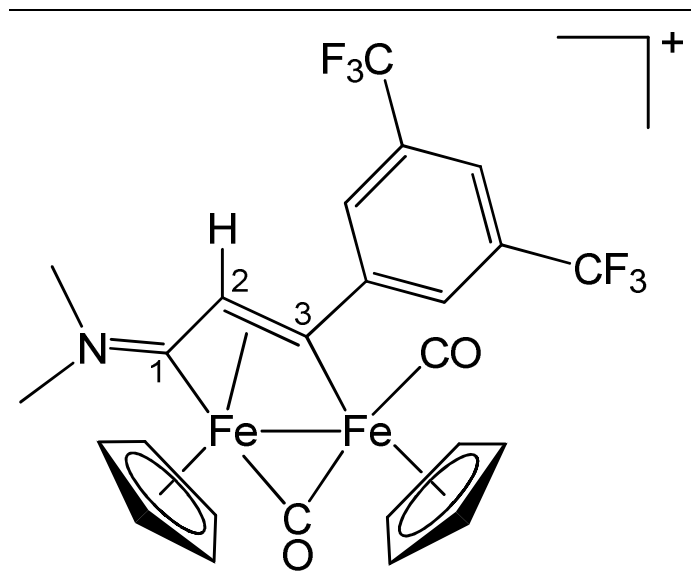

From $[1 \mathbf{c}] \mathrm{CF}_{3} \mathrm{SO}_{3}$ and 1-ethynyl-3,5-bis(trifluoromethyl)benzene. Brown solid, yield 65\%. Anal. calcd. for $\mathrm{C}_{26} \mathrm{H}_{20} \mathrm{~F}_{9} \mathrm{Fe}_{2} \mathrm{NO}_{5} \mathrm{~S}$ : C, 42.13; H, 2.72; N, 1.89. Found: C, 42.25; H, 2.60; N, 1.94. IR $\left(\mathrm{CH}_{2} \mathrm{Cl}_{2}\right)$ : $\tilde{v} / \mathrm{cm}^{-1}=1996 \mathrm{vs}(\mathrm{CO}), 1813 \mathrm{~s}(\mu-\mathrm{CO}), 1696 \mathrm{~m}-\mathrm{s}\left(\mathrm{C}^{2} \mathrm{C}^{1} \mathrm{~N}\right) .{ }^{1} \mathrm{H}$ NMR $\left(\mathrm{dmso}_{6}\right): \delta / \mathrm{ppm}=8.31,8.14(\mathrm{~m}, 3$ $\mathrm{H}$, arom); 5.40, 5.27 (s, $10 \mathrm{H}, \mathrm{Cp}) ; 4.71\left(\mathrm{~s}, 1 \mathrm{H}, \mathrm{C}^{2} \mathrm{H}\right) ; 3.86,3.42\left(\mathrm{~s}, 6 \mathrm{H}, \mathrm{NMe}_{2}\right) .{ }^{13} \mathrm{C}\left\{{ }^{1} \mathrm{H}\right\} \mathrm{NMR}(\mathrm{dmso}-$ $\left.\mathrm{d}_{6}\right): \delta / \mathrm{ppm}=256.1(\mu-\mathrm{CO}) ; 223.4\left(\mathrm{C}^{1}\right) ; 209.9(\mathrm{CO}) ; 194.8\left(\mathrm{C}^{3}\right) ; 158.4\left(\right.$ ipso- $\left.\mathrm{C}_{6} \mathrm{H}_{3}\right) ; 135.6-120.7$ (aromatics); 91.9, $88.4(\mathrm{Cp}) ; 54.0\left(\mathrm{C}^{2}\right) ; 51.8,44.8\left(\mathrm{NMe}_{2}\right) ; C \mathrm{~F}_{3}$ not observed. ${ }^{19} \mathrm{~F}\left\{{ }^{1} \mathrm{H}\right\} \mathrm{NMR}\left(\mathrm{dmso}_{6} \mathrm{~d}_{6}\right)$ : $\delta / \mathrm{ppm}=-60.8\left(\mathrm{CF}_{3}\right) ;-77.8\left(\mathrm{CF}_{3} \mathrm{SO}_{3}\right)$. Crystallization from a $\mathrm{CH}_{2} \mathrm{Cl}_{2}$ solution layered with $\mathrm{Et}_{2} \mathrm{O}$ and stored at $-30{ }^{\circ} \mathrm{C}$ afforded red-brown crystals of $\mathbf{2 i}$.

$\left[\mathrm{Fe}_{2} \mathrm{Cp}_{2}(\mathrm{CO})(\mu-\mathrm{CO})\left\{\mu-\eta^{1}: \eta^{3}-\mathrm{C}^{3}\left({ }^{\mathrm{B}} \mathrm{Bu}\right) \mathrm{C}^{2} \mathrm{HC}^{1} \mathrm{NMe}_{2}\right\}\right] \mathrm{CF}_{3} \mathrm{SO}_{3},[2 \mathrm{j}] \mathrm{CF}_{3} \mathrm{SO}_{3}$ (Chart 5).

Chart 5. Structure of $[\mathbf{2 j}]^{+}$.

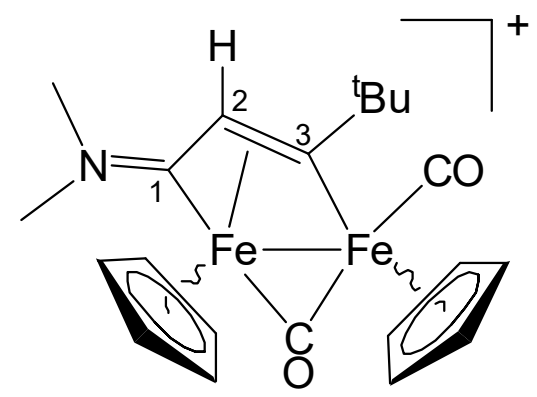


From $[\mathbf{1 c}] \mathrm{CF}_{3} \mathrm{SO}_{3}$ and 3,3-dimethyl-1-butyne. Brown-green solid, yield 90\%. Anal. calcd. for $\mathrm{C}_{22} \mathrm{H}_{26} \mathrm{~F}_{3} \mathrm{Fe}_{2} \mathrm{NO}_{5} \mathrm{~S}: \mathrm{C}, 45.15 ; \mathrm{H}, 4.48 ; \mathrm{N}, 2.39$. Found: C, 44.72; H, 4.73; N, 2.50. IR $\left(\mathrm{CH}_{2} \mathrm{Cl}_{2}\right): \tilde{\mathrm{v}} / \mathrm{cm}^{-1}=$ 1980vs (CO), 1810s $(\mu-\mathrm{CO}), 1682 \mathrm{~m}\left(\mathrm{C}^{2} \mathrm{C}^{1} \mathrm{~N}\right) .{ }^{1} \mathrm{H}$ NMR $\left(\mathrm{dmso}^{\left.-\mathrm{d}_{6}\right)}\right) \delta / \mathrm{ppm}=5.53,5.22,5.03,4.76(\mathrm{~s}$, $10 \mathrm{H}, \mathrm{Cp}) ; 4.88,4.54\left(\mathrm{~s}, 1 \mathrm{H}, \mathrm{C}^{2} \mathrm{H}\right) ; 3.92,3.81,3.16$ (s, $\left.6 \mathrm{H}, \mathrm{NMe}_{2}\right) ; 1.76,1.73\left(\mathrm{~s}, 9 \mathrm{H}, \mathrm{CMe}_{3}\right)$. trans/cis ratio $=2.5$. Crystallization from a $\mathrm{CH}_{2} \mathrm{Cl}_{2}$ solution layered with $\mathrm{Et}_{2} \mathrm{O}$ and stored at $-30{ }^{\circ} \mathrm{C}$ afforded $\mathrm{X}-$ ray quality crystals of $\mathbf{2} \mathbf{j}$.

$\left.\left[\mathrm{Fe}_{2} \mathrm{Cp}_{2}(\mathrm{CO})(\mu-\mathrm{CO})\left\{\mu-\eta^{1}: \eta^{3}-\mathrm{C}^{3}(3-\mathrm{Py}) \mathrm{C}^{2} \mathrm{HC}^{1} \mathrm{NMe}_{2}\right\}\right] \mathrm{CF}_{3} \mathrm{SO}_{3},[2]\right] \mathrm{CF}_{3} \mathrm{SO}_{3}$ (Chart 6).

Chart 6. Structure of $[21]^{+}$.

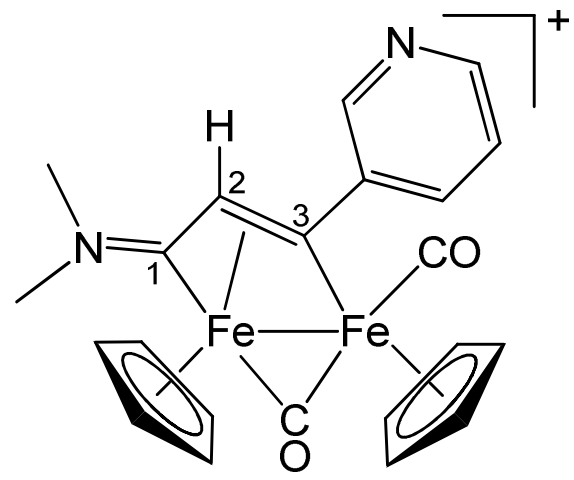

From $[1 \mathbf{c}] \mathrm{CF}_{3} \mathrm{SO}_{3}$ and 3-ethynylpyridine. Dark green solid, yield 61\%. Anal. calcd. for $\mathrm{C}_{23} \mathrm{H}_{21} \mathrm{~F}_{3} \mathrm{Fe}_{2} \mathrm{~N}_{2} \mathrm{O}_{5} \mathrm{~S}: \mathrm{C}, 45.57 ; \mathrm{H}, 3.49 ; \mathrm{N}, 4.62$. Found: C, 45.69; H, 3.55; N, 4.78. IR $\left(\mathrm{CH}_{2} \mathrm{Cl}_{2}\right): \tilde{v} / \mathrm{cm}^{-1}$ $=1994 \mathrm{vs}(\mathrm{CO}), 1809 \mathrm{~s}(\mu-\mathrm{CO}), 1683 \mathrm{~m}\left(\mathrm{C}^{2} \mathrm{C}^{1} \mathrm{~N}\right) .{ }^{1} \mathrm{H}$ NMR $\left(\mathrm{dmso}_{\mathrm{d}}\right): \delta / \mathrm{ppm}=9.00,8.68,8.22,7.60$ $\left(\mathrm{m}, 4 \mathrm{H}, \mathrm{C}_{5} \mathrm{H}_{4} \mathrm{~N}\right) ; 5.39,5.26(\mathrm{~s}, 10 \mathrm{H}, \mathrm{Cp}) ; 4.61\left(\mathrm{~s}, 1 \mathrm{H}, \mathrm{C}^{2} \mathrm{H}\right) ; 3.86,3.32\left(\mathrm{~s}, 6 \mathrm{H}, \mathrm{NMe}_{2}\right) .{ }^{13} \mathrm{C}\left\{{ }^{1} \mathrm{H}\right\} \mathrm{NMR}$ $\left(\mathrm{dmso}_{6}\right): \delta / \mathrm{ppm}=256.8(\mu-\mathrm{CO}) ; 224.0\left(\mathrm{C}^{1}\right) ; 210.2(\mathrm{CO}) ; 198.2\left(\mathrm{C}^{3}\right) ; 148.0,135.5,125.2,122.7$, $119.5\left(\mathrm{C}_{5} \mathrm{H}_{4} \mathrm{~N}\right) ; 91.9,88.3(\mathrm{Cp}) ; 53.9\left(\mathrm{C}^{2}\right) ; 51.8,44.8\left(\mathrm{NMe}_{2}\right)$.

$\left[\mathrm{Fe}_{2} \mathrm{Cp}_{2}(\mathrm{CO})(\mu-\mathrm{CO})\left\{\mu-\eta^{1}: \eta^{3}-\mathrm{C}^{3}(2-\mathrm{Py}) \mathrm{C}^{2} \mathrm{HC}^{1} \mathrm{NMe}_{2}\right\}\right] \mathrm{CF}_{3} \mathrm{SO}_{3},[2 \mathrm{n}-2] \mathrm{CF}_{3} \mathrm{SO}_{3}$ (Chart 7).

Chart 7. Structures of [2n-2] . 

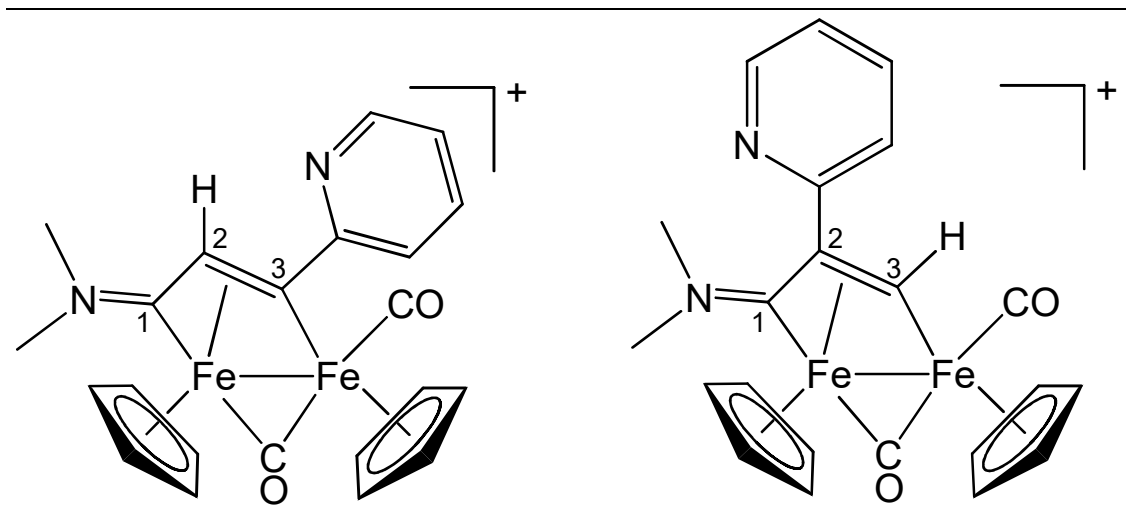

From $[\mathbf{1 c}] \mathrm{CF}_{3} \mathrm{SO}_{3}$ and 2-ethynylpyridine. Green-brown solid, yield 61\%. Anal. calcd. for $\mathrm{C}_{23} \mathrm{H}_{21} \mathrm{~F}_{3} \mathrm{Fe}_{2} \mathrm{~N}_{2} \mathrm{O}_{5} \mathrm{~S}: \mathrm{C}, 45.57 ; \mathrm{H}, 3.49 ; \mathrm{N}, 4.62$. Found: C, 45.32; H, 3.45; N, 4.50. IR $\left(\mathrm{CH}_{2} \mathrm{Cl}_{2}\right): \tilde{v} / \mathrm{cm}^{-1}$ $=1990 \mathrm{vs}(\mathrm{CO}), 1808 \mathrm{~s}(\mu-\mathrm{CO}), 1684 \mathrm{~m}\left(\mathrm{C}^{2} \mathrm{C}^{1} \mathrm{~N}\right) .{ }^{1} \mathrm{H}$ NMR $\left(\mathrm{dmso}^{-} \mathrm{d}_{6}\right): \delta / \mathrm{ppm}=12.84\left(\mathrm{~s}, 1 \mathrm{H}, \mathrm{C}^{3} \mathrm{H}\right)$; 8.76, 7.97, 7.79, $7.43\left(\mathrm{~m}, 4 \mathrm{H}, \mathrm{C}_{5} \mathrm{H}_{4} \mathrm{~N}\right) ; 5.60,5.29,5.19,4.92(\mathrm{~s}, 10 \mathrm{H}, \mathrm{Cp}) ; 4.71\left(\mathrm{C}^{2} \mathrm{H}\right) ; 3.83,3.28(\mathrm{~s}, 6$ $\left.\mathrm{H}, \mathrm{NMe}_{2}\right)$. Isomer ratio $=$ ca. $5 .{ }^{13} \mathrm{C}\left\{{ }^{1} \mathrm{H}\right\}$ NMR $\left(\mathrm{dmso}_{6}\right): \delta / \mathrm{ppm}=257.1(\mu-\mathrm{CO}) ; 224.4\left(\mathrm{C}^{1}\right) ; 210.6$ (CO); 200.2, $171.7\left(\mathrm{C}^{3}\right)$; 149.3, 137.3, 122.6, $122.4\left(\mathrm{C}_{5} \mathrm{H}_{4} \mathrm{~N}\right)$; 91.4, 90.8, 88.4, 88.1 (Cp); $52.7\left(\mathrm{C}^{2}\right)$; $51.4,44.9\left(\mathrm{NMe}_{2}\right)$

\section{2) Synthesis and characterization of 3a-m.}

General procedure. The appropriate precursor $[2] \mathrm{CF}_{3} \mathrm{SO}_{3}$ (ca. $0.5 \mathrm{mmol}$ ) was dissolved in tetrahydrofuran $(15-20 \mathrm{~mL})$, then pyrrolidine (ca. 10 eq.) was added. The mixture was stirred at room temperature overnight, then it was passed through a short alumina pad using neat acetonitrile as eluent. The filtrated solution was dried under vacuum. The residue was dissolved in diethyl ether or diethyl ether/dichloromethane mixture and charged on alumina column. Elution with petroleum ether/diethyl ether mixtures allowed any impurities to be removed, then the fraction corresponding to the desired product was collected. Removal of the solvent under reduced pressure afforded an air stable solid. Yields are given with respect to $\mathrm{C}^{1}$. 
Chart 8. Structure of 3a.

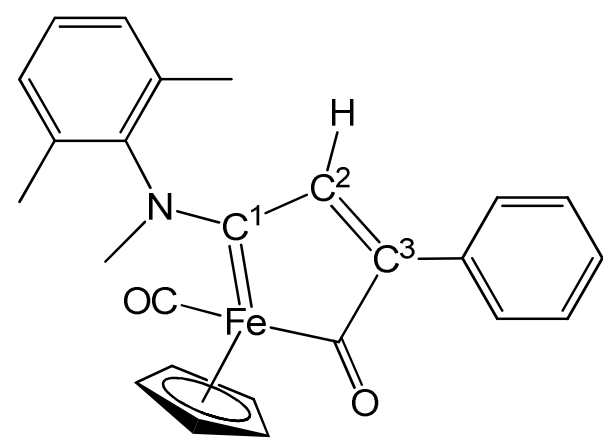

From $[\mathbf{2 a}] \mathrm{CF}_{3} \mathrm{SO}_{3}$. Brown solid, yield 93\%. Eluent for chromatography: $\mathrm{Et}_{2} \mathrm{O}$. Anal. calcd. for $\mathrm{C}_{25} \mathrm{H}_{23} \mathrm{FeNO}_{2}$ : C, 70.60; H, 5.45; N, 3.29. Found: C, 70.50; H, 5.48; N, 3.21. IR $\left(\mathrm{CH}_{2} \mathrm{Cl}_{2}\right): \tilde{v} / \mathrm{cm}^{-1}=$ 1919vs (CO), 1612m (CO $\left.\mathrm{CO}_{\text {acyl }}\right), 1571 \mathrm{~m}$. IR (solid state): $\tilde{\mathrm{v}} / \mathrm{cm}^{-1}=3082 \mathrm{w}, 3029 \mathrm{w}, 2925 \mathrm{w}, 1907 \mathrm{vs}$, $1614 \mathrm{~m}\left(\mathrm{CO}_{\text {acyl }}\right), 1571 \mathrm{w}-\mathrm{m}\left(\mathrm{C}^{1} \mathrm{~N}\right), 1471 \mathrm{~m}, 1383 \mathrm{~m}, 1352 \mathrm{w}, 1299 \mathrm{w}, 1084 \mathrm{~s}-\mathrm{br}, 1015 \mathrm{~s}-\mathrm{br}, 879 \mathrm{w}, 798 \mathrm{vs}$, 774vs, 719w, 696m, 656w. ${ }^{1} \mathrm{H}$ NMR (acetone-d 6 ): $\delta / \mathrm{ppm}=7.38-7.22\left(\mathrm{~m}, 8 \mathrm{H}, \mathrm{C}_{6} \mathrm{H}_{3} \mathrm{Me}_{2}+\mathrm{Ph}\right) ; 6.96(\mathrm{~s}$, $\left.1 \mathrm{H}, \mathrm{C}^{2} \mathrm{H}\right) ; 4.73(\mathrm{~s}, 5 \mathrm{H}, \mathrm{Cp}) ; 3.96$ (s, $\left.3 \mathrm{H}, \mathrm{NMe}\right) ; 2.32,2.17\left(\mathrm{~s}, 6 \mathrm{H}, \mathrm{C}_{6} \mathrm{H}_{3} M e_{2}\right) .{ }^{20}{ }^{13} \mathrm{C}\left\{{ }^{1} \mathrm{H}\right\} \mathrm{NMR}$ $\left(\right.$ acetone- $\left.\mathrm{d}_{6}\right): \delta / \mathrm{ppm}=264.7,264.6\left(\mathrm{CO}_{\text {acyl }}+\mathrm{C}^{1}\right) ; 221.9(\mathrm{CO}) ; 168.7\left(\mathrm{C}^{3}\right) ; 147.5\left(\mathrm{C}^{2}\right) ; 145.5$ (ipso$\left.\mathrm{C}_{6} \mathrm{H}_{3}\right) ; 132.9,132.7,132.4,129.1,129.0,128.9,128.8,128.7,127.9\left(C_{6} \mathrm{H}_{3} \mathrm{Me}_{2}+\mathrm{Ph}\right) ; 85.1(\mathrm{Cp}) ; 48.8$ $(\mathrm{NMe}) ; 17.0,16.6\left(\mathrm{C}_{6} \mathrm{H}_{3} M e_{2}\right){ }^{20}$

\section{$\left[\mathrm{FeCp}(\mathrm{CO})\left\{\mathrm{C}^{1} \mathrm{~N}(\mathrm{Me})(\mathrm{Xyl}) \mathrm{C}^{2} \mathrm{HC}^{3}\left({ }^{\mathrm{t}} \mathrm{Bu}\right) \mathrm{C}(=\mathrm{O})\right\}\right], 3 \mathrm{~b}\left(\mathrm{Chart}^{9}\right)$.}

\section{Chart 9. Structure of $\mathbf{3 b}$.}

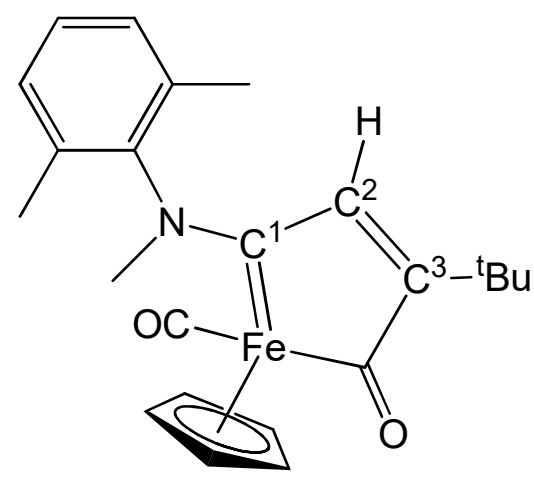


From $[\mathbf{2 b}] \mathrm{CF}_{3} \mathrm{SO}_{3}$. Brown solid, yield 40\%. Eluent for chromatography: $\mathrm{Et}_{2} \mathrm{O} / \mathrm{CH}_{2} \mathrm{Cl}_{2}$ 1:1 v/v (quick chromatography). Anal. calcd. for $\mathrm{C}_{22} \mathrm{H}_{29} \mathrm{FeNO}_{2}$ : C, 66.84; H, 7.39; N, 3.54. Found: C, 66.70; H, 7.46; $\mathrm{N}, 3.61 . \mathrm{IR}\left(\mathrm{CH}_{2} \mathrm{Cl}_{2}\right): \tilde{\mathrm{v}} / \mathrm{cm}^{-1}=1913 \mathrm{vs}(\mathrm{CO}), 1616 \mathrm{~m}\left(\mathrm{CO}_{\text {acyl }}\right), 1598 \mathrm{w}$. IR (solid state): $\tilde{\mathrm{v}} / \mathrm{cm}^{-1}=2907 \mathrm{w}$, 1915m-sh, 1906vs (CO), 1614m (CO acyl $\left._{1}\right), 1599 \mathrm{w}-\mathrm{m}, 1568 \mathrm{w}, 1477 \mathrm{~m}, 1429 \mathrm{w}-\mathrm{m}, 1381 \mathrm{~m}, 1359 \mathrm{w}-\mathrm{m}$, 1243w, 1143w, 1087m, 1068m-s, 1014m-s, 881w, 854m, 803vs, 744w, 724m, 709m, 688w, 658w. ${ }^{1} \mathrm{H}$ NMR $\left(\right.$ dmso-d $\left._{6}\right): \delta / p p m=7.29-7.24\left(\mathrm{~m}, 3 \mathrm{H}, \mathrm{C}_{6} H_{3} \mathrm{Me}_{2}\right) ; 6.45\left(\mathrm{~s}, 1 \mathrm{H}, \mathrm{C}^{2} \mathrm{H}\right) ; 4.60(\mathrm{~s}, 5 \mathrm{H}, \mathrm{Cp}) ; 3.78$ (s, $3 \mathrm{H}, \mathrm{NMe}) ; 2.18,2.03\left(\mathrm{~s}, 6 \mathrm{H}, \mathrm{C}_{6} \mathrm{H}_{3} M e_{2}\right) ; 0.92\left(\mathrm{~s}, 9 \mathrm{H}, \mathrm{CMe}_{3}\right) \cdot{ }^{13} \mathrm{C}\left\{{ }^{1} \mathrm{H}\right\} \mathrm{NMR}\left(\mathrm{dmso}_{6}\right): \delta / \mathrm{ppm}=$ $267.8\left(\mathrm{CO}_{\text {acyl }}\right) ; 264.4\left(\mathrm{C}^{1}\right) ; 222.6(\mathrm{CO}) ; 183.6\left(\mathrm{C}^{3}\right) ; 146.3\left(\mathrm{C}^{2}\right) ; 145.4$ (ipso- $\left.C_{6} \mathrm{H}_{3} \mathrm{Me}_{2}\right) ; 132.6$, 132.4, 129.3, $129.1\left(C_{6} \mathrm{H}_{3} \mathrm{Me}_{2}\right) ; 85.5(\mathrm{Cp}) ; 49.1(\mathrm{NMe}) ; 34.2\left(\mathrm{CMe}_{3}\right) ; 29.1\left(\mathrm{CMe}_{3}\right) ; 17.7,17.2\left(\mathrm{C}_{6} \mathrm{H}_{3} M e_{2}\right)$. Crystals of $\mathbf{3 b}$ suitable for X-ray analysis were collected from a diethyl ether solution layered with pentane, stored at $-30{ }^{\circ} \mathrm{C}$.

\section{$\left[\mathrm{FeCp}(\mathrm{CO})\left\{\mathrm{C}^{1} \mathrm{~N}(\mathrm{Me})(\mathrm{Xyl}) \mathrm{C}^{2} \mathrm{HC}^{3}(2-\right.\right.$ naphthyl $\left.\left.) \mathrm{C}(=\mathbf{O})\right\}\right], 3 \mathrm{c}$ (Chart 10).}

\section{Chart 10. Structure of 3c.}

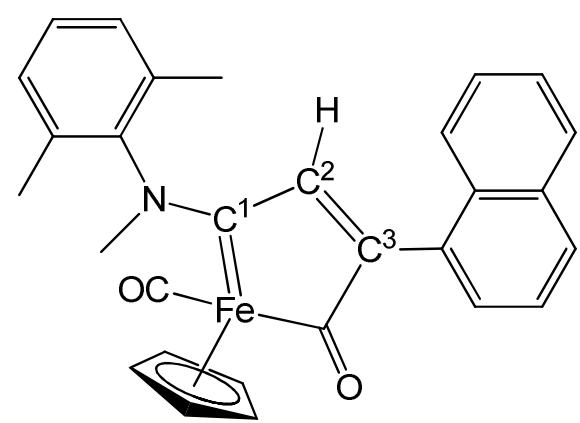

From $[\mathbf{2 c}] \mathrm{CF}_{3} \mathrm{SO}_{3}$. Brown solid, yield 55\%. Eluent for chromatography: $\mathrm{Et}_{2} \mathrm{O} / \mathrm{CH}_{2} \mathrm{Cl}_{2}$ 1:1 v/v. Anal. calcd. for $\mathrm{C}_{29} \mathrm{H}_{25} \mathrm{FeNO}_{2}$ : C, 73.27; H, 5.30; N, 2.95. Found: C, 73.10; H, 5.41; N, 3.06. IR $\left(\mathrm{CH}_{2} \mathrm{Cl}_{2}\right)$ : $\tilde{\mathrm{v}} / \mathrm{cm}^{-1}=1920 \mathrm{vs}(\mathrm{CO}), 1610 \mathrm{~s}\left(\mathrm{CO}_{\text {acyl }}\right)$. IR (solid state) $\tilde{\mathrm{v}} / \mathrm{cm}^{-1}=2922 \mathrm{vw}, 1908 \mathrm{vs}(\mathrm{CO}), 1610 \mathrm{~m}$ $\left(\mathrm{CO}_{\text {acyl }}\right), 1558 \mathrm{w}, 1486 \mathrm{~m}, 1434 \mathrm{w}-\mathrm{m}, 1388 \mathrm{w}-\mathrm{m}, 1353 \mathrm{w}, 1238 \mathrm{w}, 1164 \mathrm{w}, 1135 \mathrm{w}-\mathrm{m}, 1079 \mathrm{vs}, 1018 \mathrm{~s}$, 959w, 846w, 801vs, 734w, 712w, 658w. ${ }^{1} \mathrm{H}$ NMR $\left(\mathrm{dmso}_{6}\right): \delta / \mathrm{ppm}=7.88,7.46-7.39,7.23,7.02(\mathrm{~m}$, 
$\left.10 \mathrm{H}, \mathrm{C}_{10} \mathrm{H}_{7}+\mathrm{C}_{6} \mathrm{H}_{3} \mathrm{Me}_{2}\right) ; 6.72\left(\mathrm{~s}, 1 \mathrm{H}, \mathrm{C}^{2} \mathrm{H}\right) ; 4.80(\mathrm{~s}, 5 \mathrm{H}, \mathrm{Cp}) ; 3.90$ (s, $\left.3 \mathrm{H}, \mathrm{NMe}\right) ; 2.27,2.15(\mathrm{~s}, 6 \mathrm{H}$, $\left.\mathrm{C}_{6} \mathrm{H}_{3} M e_{2}\right) .{ }^{13} \mathrm{C}\left\{{ }^{1} \mathrm{H}\right\}$ NMR $\left(\mathrm{dmso}_{6}\right): \delta / \mathrm{ppm}=264.7,263.2\left(\mathrm{CO}_{\text {acyl }}+\mathrm{C}^{1}\right) ; 222.5(\mathrm{CO}) ; 171.2\left(\mathrm{C}^{3}\right) ;$ $150.5\left(\mathrm{C}^{2}\right) ; 145.5$ (ipso- $\left.\mathrm{C}_{6} \mathrm{H}_{3} \mathrm{Me}_{2}\right)$; 132.9, 132.8, 132.3, 131.2, 129.5, 129.4, 129.2, 128.7, 128.6, 126.5, 126.4, 126.3, 125.9, $125.5\left(\mathrm{C}_{10} \mathrm{H}_{7}+C_{6} \mathrm{H}_{3} \mathrm{Me}_{2}\right) ; 85.7(\mathrm{Cp}) ; 49.5(\mathrm{NMe}) ; 17.7,17.3\left(\mathrm{C}_{6} \mathrm{H}_{3} M e_{2}\right)$.

\section{$\left[\mathrm{FeCp}(\mathrm{CO})\left\{\mathrm{C}^{1} \mathrm{~N}(\mathrm{Me})(\mathrm{Xyl}) \mathrm{C}^{2} \mathrm{HC}^{3}(3-p y r i d i n e) C(=0)\right\}\right], 3 d$ (Chart 11).}

Chart 11. Structure of $\mathbf{3 d}$.

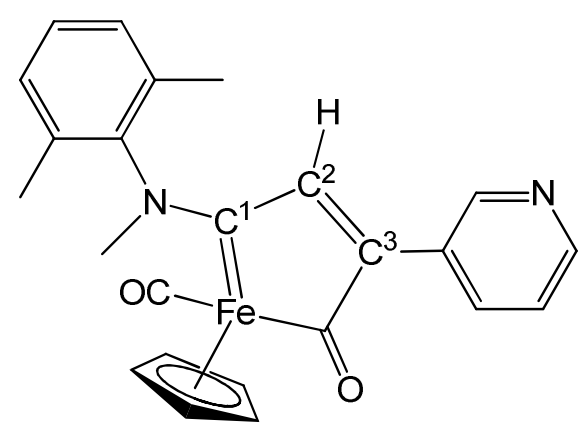

From [2d] $\mathrm{CF}_{3} \mathrm{SO}_{3}$. Brown solid, yield $65 \%$. Eluent for chromatography: $\mathrm{CH}_{2} \mathrm{Cl}_{2}$. Anal. calcd. for $\mathrm{C}_{24} \mathrm{H}_{22} \mathrm{FeN}_{2} \mathrm{O}_{2}$ : C, 67.62; H, 5.20; N, 6.57. Found: C, 67.51; H, 5.28; N, 6.49. IR $\left(\mathrm{CH}_{2} \mathrm{Cl}_{2}\right): \tilde{v} / \mathrm{cm}^{-1}=$ 1922vs (CO), 1607m (CO $\left.\mathrm{CO}_{\text {acyl }}\right), 1564 \mathrm{w}$. IR (solid state): $\tilde{\mathrm{v}} / \mathrm{cm}^{-1}=1914 \mathrm{vs}(\mathrm{CO}), 1601 \mathrm{~m}\left(\mathrm{CO}_{\text {acyl }}\right), 1561 \mathrm{w}$, $1489 \mathrm{~m}, 1471 \mathrm{~m}, 1438 \mathrm{w}, 1416 \mathrm{w}, 1394 \mathrm{~m}, 1262 \mathrm{~m}, 1241 \mathrm{~m}, 1187 \mathrm{~m}, 1168 \mathrm{~m}, 1089 \mathrm{vs}, 1139 \mathrm{~m}-\mathrm{s}, 1053 \mathrm{~s}$, $1025 \mathrm{~s}, 986 \mathrm{~m}, 920 \mathrm{w}, 885 \mathrm{w}-\mathrm{m}, 840 \mathrm{w}-\mathrm{m}, 816 \mathrm{~s}, 804 \mathrm{~s}, 703 \mathrm{~m}-\mathrm{s}, 682 \mathrm{~m}, 655 \mathrm{~m} .{ }^{1} \mathrm{H}$ NMR $\left(\mathrm{dmso}_{\mathrm{m}}\right): \delta / \mathrm{ppm}$ $=8.42,7.64,7.29\left(\mathrm{~m}, 7 \mathrm{H}, \mathrm{C}_{5} \mathrm{H}_{4} \mathrm{~N}+\mathrm{C}_{6} H_{3} \mathrm{Me}_{2}\right) ; 6.89\left(\mathrm{~s}, 1 \mathrm{H}, \mathrm{C}^{2} \mathrm{H}\right) ; 4.74(\mathrm{~s}, 5 \mathrm{H}, \mathrm{Cp}) ; 3.86(\mathrm{~s}, 3 \mathrm{H}$, $\mathrm{NMe}) ; 2.24,2.10\left(\mathrm{~s}, 6 \mathrm{H}, \mathrm{C}_{6} \mathrm{H}_{3} M e_{2}\right) .{ }^{13} \mathrm{C}\left\{{ }^{1} \mathrm{H}\right\} \mathrm{NMR}\left(\mathrm{dmso}_{6}\right): \delta / \mathrm{ppm}=266.1\left(\mathrm{CO}_{\text {acyl }}\right) ; 262.9\left(\mathrm{C}^{1}\right)$; $222.3(\mathrm{CO}) ; 165.5\left(\mathrm{C}^{3}\right) ; 150.0,149.2,145.4,136.5,132.8,132.4,129.5,129.4,129.2,128.5,123.8$ $\left(\mathrm{C}_{5} \mathrm{H}_{4} \mathrm{~N}+\mathrm{C}_{6} \mathrm{H}_{3} \mathrm{Me}_{2}\right) ; 148.3\left(\mathrm{C}^{2}\right) ; 85.7(\mathrm{Cp}) ; 49.7(\mathrm{NMe}) ; 17.7,17.3\left(\mathrm{C}_{6} \mathrm{H}_{3} M e_{2}\right)$.

$\left[\mathrm{FeCp}(\mathrm{CO})\left\{\mathrm{C}^{1} \mathrm{~N}(\mathrm{Me})(\mathrm{Xyl}) \mathrm{C}^{2} \mathrm{HC}^{3}(2\right.\right.$-pyridine $\left.\left.) \mathrm{C}(=\mathrm{O})\right\}\right]$, 3e (Chart 12).

Chart 12. Structure of $3 \mathrm{e}$. 


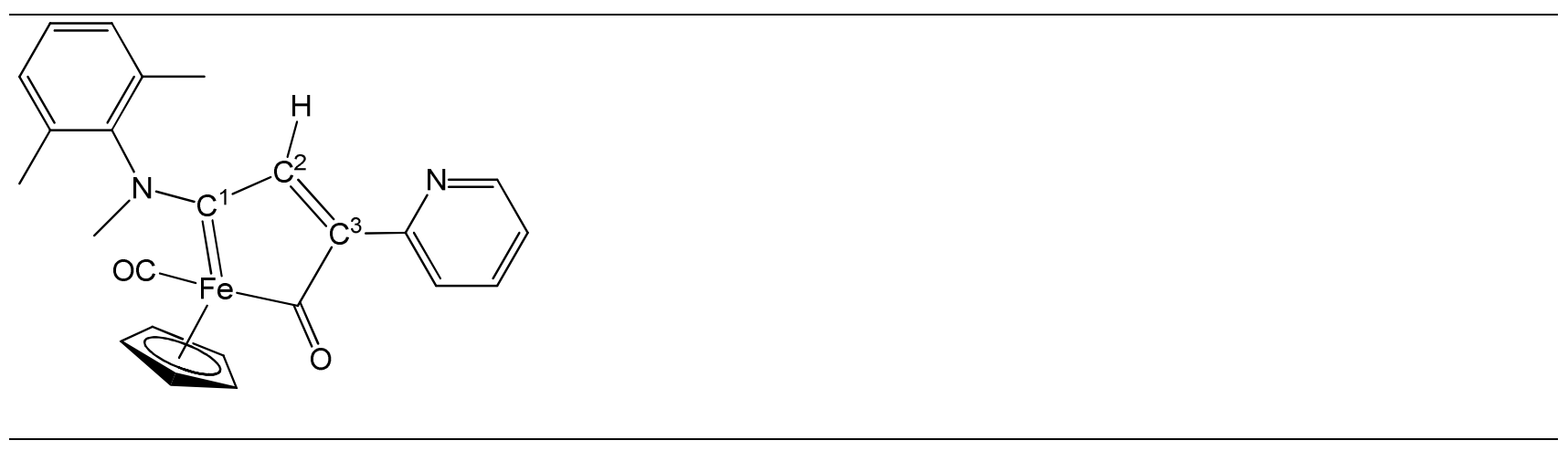

From $[2 \mathrm{e}] \mathrm{CF}_{3} \mathrm{SO}_{3}$. Red solid, yield $68 \%$. Eluent for chromatography: $\mathrm{CH}_{2} \mathrm{Cl}_{2}$. Anal. calcd. for $\mathrm{C}_{24} \mathrm{H}_{22} \mathrm{FeN}_{2} \mathrm{O}_{2}$ : C, 67.62; H, 5.20; N, 6.57. Found: C, 67.41; H, 5.23; N, 6.64. IR $\left(\mathrm{CH}_{2} \mathrm{Cl}_{2}\right): \tilde{v} / \mathrm{cm}^{-1}=$ 1919vs (CO), 1608s (CO $\left.\mathrm{CO}_{\text {acyl }}\right), 1561 \mathrm{~m}$. IR (solid state): $\tilde{v} / \mathrm{cm}^{-1}=3075 \mathrm{w}, 3029 \mathrm{w}, 2944 \mathrm{w}, 2924 \mathrm{w}, 1916 \mathrm{vs}$ (CO), 1605m ( $\left.\mathrm{CO}_{\text {acyl }}\right), 1578 \mathrm{w}-\mathrm{m}, 1558 \mathrm{w}-\mathrm{m}, 1494 \mathrm{~m}, 1459 \mathrm{w}-\mathrm{m}, 1426 \mathrm{~m}, 1391 \mathrm{~m}, 1383 \mathrm{~m}, 1273 \mathrm{w}$, $1237 \mathrm{w}, 1140 \mathrm{w}, 1083 \mathrm{~m}, 1054 \mathrm{w}, 1007 \mathrm{~m}, 986 \mathrm{w}-\mathrm{m}, 891 \mathrm{w}, 843 \mathrm{w}, 818 \mathrm{w}-\mathrm{m}, 809 \mathrm{w}-\mathrm{m}, 789 \mathrm{~s}, 779 \mathrm{~s}, 741 \mathrm{~m}$, $723 \mathrm{w}, 697 \mathrm{w}, 682 \mathrm{w}, 654 \mathrm{w} .{ }^{1} \mathrm{H}$ NMR $\left(\mathrm{dmso}^{-} \mathrm{d}_{6}\right): \delta / \mathrm{ppm}=8.44,7.85,7.74,7.30\left(\mathrm{~m}, 7 \mathrm{H}, \mathrm{C}_{5} \mathrm{H}_{4} \mathrm{~N}+\right.$ $\mathrm{C}_{6} \mathrm{H}_{3} \mathrm{Me}_{2}$ ); 7.38 (s, $\left.1 \mathrm{H}, \mathrm{C}^{2} \mathrm{H}\right) ; 4.74$ (s, $\left.5 \mathrm{H}, \mathrm{Cp}\right) ; 3.83$ (s, $\left.3 \mathrm{H}, \mathrm{NMe}\right) ; 2.22,2.08$ (s, $\left.6 \mathrm{H}, \mathrm{C}_{6} \mathrm{H}_{3} M e_{2}\right)$. ${ }^{13} \mathrm{C}\left\{{ }^{1} \mathrm{H}\right\}$ NMR $\left(\mathrm{dmso} \mathrm{d}_{6}\right): \delta / \mathrm{ppm}=267.3\left(\mathrm{CO}_{\mathrm{acyl}}\right) ; 262.8\left(\mathrm{C}^{1}\right) ; 222.6(\mathrm{CO}) ; 167.0\left(\mathrm{C}^{3}\right) ; 150.4,150.1$, 145.6, 136.5, 132.8, 132.4, 129.6, 129.5, 129.2, 125.8, $124.5\left(\mathrm{C}_{5} \mathrm{H}_{4} \mathrm{~N}+C_{6} \mathrm{H}_{3} \mathrm{Me}_{2}\right) ; 149.9\left(\mathrm{C}^{2}\right), 85.8$ (Cp); 49.7 (NMe); 17.7, $17.3\left(\mathrm{C}_{6} \mathrm{H}_{3} M e_{2}\right)$.

$\left[\mathrm{FeCp}(\mathrm{CO})\left\{\mathrm{C}^{1} \mathrm{~N}(\mathrm{Me})(\mathrm{Xyl}) \mathrm{C}^{2} \mathrm{HC}^{3}\right.\right.$ (3-thiophene)C(=O) $\left.\}\right]$, 3f (Chart 13).

Chart 13. Structure of 3f.

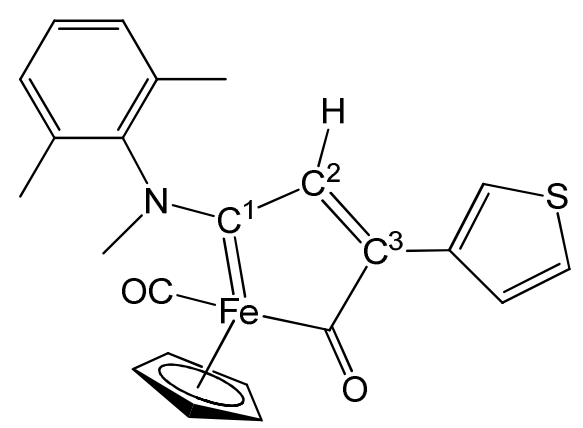


From [2f] $\mathrm{CF}_{3} \mathrm{SO}_{3}$. Brown solid, yield $70 \%$. Eluent for chromatography: $\mathrm{CH}_{2} \mathrm{Cl}_{2}$. Anal. calcd. for $\mathrm{C}_{23} \mathrm{H}_{21} \mathrm{FeNO}_{2} \mathrm{~S}: \mathrm{C}, 64.05 ; \mathrm{H}, 4.91 ; \mathrm{N}, 3.25$. Found: $\mathrm{C}, 63.91 ; \mathrm{H}, 4.98 ; \mathrm{N}, 3.35$. IR $\left(\mathrm{CH}_{2} \mathrm{Cl}_{2}\right): \tilde{v} / \mathrm{cm}^{-1}=$ 1918vs (CO), 1615m (CO acyl), 1596s. IR (solid state): $\tilde{v} / \mathrm{cm}^{-1}=3075 \mathrm{w}, 3030 \mathrm{vw}, 2944 \mathrm{w}, 1908 \mathrm{vs}(\mathrm{CO})$, $1616 \mathrm{~m}-\mathrm{sh}, 1594 \mathrm{~m}-\mathrm{s}, 1483 \mathrm{~m}, 1471 \mathrm{~m}, 1441 \mathrm{w}, 1418 \mathrm{w}, 1387 \mathrm{~m}, 1380 \mathrm{~m}, 1364 \mathrm{w}, 1352 \mathrm{w}, 1284 \mathrm{w}, 1263 \mathrm{w}$, $1238 \mathrm{w}, 1140 \mathrm{w}-\mathrm{m}, 1085 \mathrm{w}-\mathrm{m}, 1074 \mathrm{w}-\mathrm{m}, 1050 \mathrm{w}, 1003 \mathrm{~m}, 900 \mathrm{w}, 885 \mathrm{w}, 873 \mathrm{w}, 845 \mathrm{w}, 823 \mathrm{~m}, 799 \mathrm{~s}, 716 \mathrm{w}-$ m, 696m. ${ }^{1} \mathrm{H}$ NMR $\left(\mathrm{dmso}^{\mathrm{d}}{ }_{6}\right): \delta / \mathrm{ppm}=8.04,7.43,7.34-7.25,6.96\left(\mathrm{~m}, 6 \mathrm{H}, \mathrm{C}_{4} \mathrm{H}_{3} \mathrm{~S}+\mathrm{C}_{6} H_{3} \mathrm{Me}_{2}\right) ; 6.86$ $\left(\mathrm{s}, 1 \mathrm{H}, \mathrm{C}^{2} \mathrm{H}\right) ; 4.70(\mathrm{~s}, 5 \mathrm{H}, \mathrm{Cp}) ; 3.81(\mathrm{~s}, 3 \mathrm{H}, \mathrm{NMe}) ; 2.22,2.07\left(\mathrm{~s}, 6 \mathrm{H} ; \mathrm{C}_{6} \mathrm{H}_{3} M e_{2}\right) .{ }^{13} \mathrm{C}\left\{{ }^{1} \mathrm{H}\right\} \mathrm{NMR}$ $\left(\mathrm{dmso}_{6}\right): \delta / \mathrm{ppm}=267.3\left(\mathrm{CO}_{\text {acyl }}\right), 261.8\left(\mathrm{C}^{1}\right) ; 222.6(\mathrm{CO}) ; 162.1\left(\mathrm{C}^{3}\right) ; 150.0\left(\mathrm{C}^{2}\right) ; 145.5$ (ipso$\left.\mathrm{C}_{6} \mathrm{H}_{3} \mathrm{Me}_{2}\right) ; 133.1,132.9,132.4,129.5,129.4,129.1,127.9,126.7\left(\mathrm{C}_{4} \mathrm{H}_{3} \mathrm{~S}+C_{6} \mathrm{H}_{3} \mathrm{Me}_{2}\right) ; 85.6(\mathrm{Cp}) ; 49.4$ (NMe); 17.7, $17.3\left(\mathrm{C}_{6} \mathrm{H}_{3} M e_{2}\right)$. Crystals of $\mathbf{3 f}$ suitable for $\mathrm{X}$-ray analysis were collected from a dichloromethane solution layered with pentane, stored at $-30^{\circ} \mathrm{C}$.

\section{$\left[\mathrm{FeCp}(\mathrm{CO})\left\{\mathrm{C}^{1} \mathrm{~N}(\mathrm{Me})\left(\mathrm{Xyl}^{\mathrm{Cl}}\right) \mathrm{C}^{2} \mathrm{HC}^{3}(\mathrm{Ph}) \mathrm{C}(=\mathrm{O})\right\}\right], 3 \mathrm{~g}(\mathrm{Chart} 14)$.}

\section{Chart 14. Structure of $3 g$.}

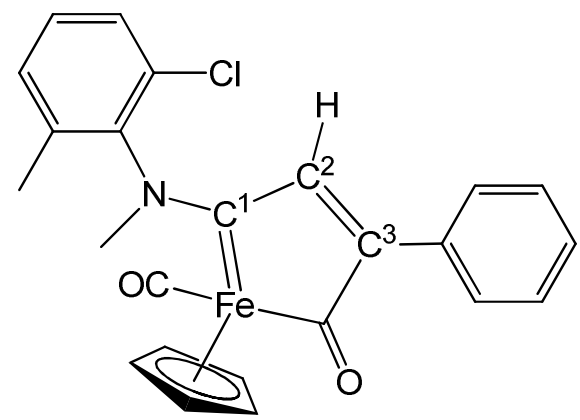

From $[\mathbf{2 g}] \mathrm{CF}_{3} \mathrm{SO}_{3}$. Brown solid, yield 75\%. Eluent for chromatography: $\mathrm{CH}_{2} \mathrm{Cl}_{2} / \mathrm{Et}_{2} \mathrm{O}$ 1:1 v/v. Anal. calcd. for $\mathrm{C}_{24} \mathrm{H}_{20} \mathrm{ClFeNO}_{2}: \mathrm{C}, 64.67 ; \mathrm{H}, 4.52 ; \mathrm{N}, 3.14$. Found: $\mathrm{C}, 64.50 ; \mathrm{H}, 4.64 ; \mathrm{N}, 3.06 . \mathrm{IR}\left(\mathrm{CH}_{2} \mathrm{Cl}_{2}\right)$ : $\tilde{\mathrm{v}} / \mathrm{cm}^{-1}=1922 \mathrm{vs}(\mathrm{CO}), 1615 \mathrm{~m}\left(\mathrm{CO}_{\text {acyl }}\right), 1571 \mathrm{w}$. IR (solid state) $\tilde{\mathrm{v}} / \mathrm{cm}^{-1}=3078 \mathrm{vw}, 2964 \mathrm{w}-\mathrm{m}, 1912 \mathrm{~m}-\mathrm{s}$ (CO), 1615m ( $\left.\mathrm{CO}_{\text {acyl }}\right), 1570 \mathrm{w}, 1477 \mathrm{w}-\mathrm{m}, 1382 \mathrm{w}-\mathrm{m}, 1302 \mathrm{w}, 1260 \mathrm{~s}, 1243 \mathrm{w}-\mathrm{m}, 1186 \mathrm{w}, 1161 \mathrm{w}, 1079 \mathrm{~s}$, 1015vs, 989s, 886m, 865m, 848m, 796vs, 779vs, 714m, 700m, 693m-s, 667m. ${ }^{1} \mathrm{H} \mathrm{NMR}\left(\mathrm{CDCl}_{3}\right)$ : 
$\delta / \mathrm{ppm}=7.45-7.26\left(\mathrm{~m}, 8 \mathrm{H}, \mathrm{C}_{6} \mathrm{H}_{3} \mathrm{ClMe}+\mathrm{Ph}\right) ; 6.88,6.85\left(\mathrm{~s}, 1 \mathrm{H}, \mathrm{C}^{2} \mathrm{H}\right) ; 4.73,4.72(\mathrm{~s}, 5 \mathrm{H}, \mathrm{Cp}) ; 3.91$, 3.90 (s, $3 \mathrm{H}, \mathrm{NMe}) ; 2.31,2.21\left(\mathrm{C}_{6} \mathrm{H}_{3} \mathrm{Cl} M e\right)$. Isomer ratio $=1 .{ }^{13} \mathrm{C}\left\{{ }^{1} \mathrm{H}\right\} \mathrm{NMR}\left(\mathrm{CDCl}_{3}\right): \delta / \mathrm{ppm}=269.0$, 268.6, 268.3, 267.9 $\left(\mathrm{CO}_{\text {acyl }}+\mathrm{C}^{1}\right) ; 221.0(\mathrm{CO}) ; 170.0,169.8\left(\mathrm{C}^{3}\right) ; 147.5,147.4\left(\mathrm{C}^{2}\right)$; 143.3, 143.0 (ipso$\left.\mathrm{C}_{6} \mathrm{H}_{3} \mathrm{ClMe}\right) ; 135.5,135.0,132.5,130.1,129.8,129.7,129.6,129.2,129.1,128.5,128.2,128.1$ $\left(C_{6} \mathrm{H}_{3} \mathrm{ClMe}+\mathrm{Ph}\right) ; 85.4,85.3(\mathrm{Cp}) ; 48.9,48.6(\mathrm{NMe}) ; 18.1,17.9\left(\mathrm{C}_{6} \mathrm{H}_{3} \mathrm{ClMe}\right)$. Crystallization from a $\mathrm{CH}_{2} \mathrm{Cl}_{2}$ solution layered with pentane and stored at $-30{ }^{\circ} \mathrm{C}$ afforded dark-red crystals of $\mathbf{3 g}$.

$\left[\mathrm{FeCp}(\mathrm{CO})\left\{\mathrm{C}^{1} \mathrm{~N}(\mathrm{Me})_{2} \mathrm{C}^{2} \mathrm{HC}^{3}(\mathrm{Ph}) \mathrm{C}(=\mathrm{O})\right\}\right], 3 \mathrm{~h}(\mathrm{Chart} 15)$.

Chart 15. Structure of $3 \mathrm{~h}$.

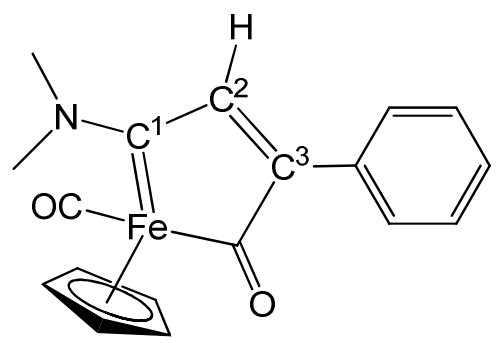

From $[\mathbf{2 h}] \mathrm{CF}_{3} \mathrm{SO}_{3}$. Dark-red solid, yield 66\%. Eluent for chromatography: $\mathrm{CH}_{2} \mathrm{Cl}_{2} / \mathrm{THF}$ 1:1 v/v. Anal. calcd. for $\mathrm{C}_{18} \mathrm{H}_{17} \mathrm{FeNO}_{2}$ : C, 64.50; H, 5.11; N, 4.18. Found: C, 64.36; H, 5.23; N, 4.26. IR $\left(\mathrm{CH}_{2} \mathrm{Cl}_{2}\right)$ : $\tilde{\mathrm{v}} / \mathrm{cm}^{-1}=1915 \mathrm{vs}(\mathrm{CO}), 1602 \mathrm{~m}\left(\mathrm{CO}_{\text {acyl }}\right), 1526 \mathrm{~m}$. IR (solid state): $\tilde{\mathrm{v}} / \mathrm{cm}^{-1}=3104 \mathrm{vw}, 2969 \mathrm{vw}, 2941 \mathrm{vw}$, $1893 \mathrm{vs}(\mathrm{CO}), 1588 \mathrm{~s}\left(\mathrm{CO}_{\text {acyl }}\right), 1524 \mathrm{~m}-\mathrm{s}, 1488 \mathrm{w}, 1442 \mathrm{w}-\mathrm{m}, 1409 \mathrm{~m}, 1398 \mathrm{~m}, 1356 \mathrm{w}, 1320 \mathrm{w}, 1299 \mathrm{w}$, 1260w, 1238w-m, 1177w-m, 1150w-m, 1114w-m, 1088w-m, 1073w-m, 1030m, 1006m, 869m, 845w-

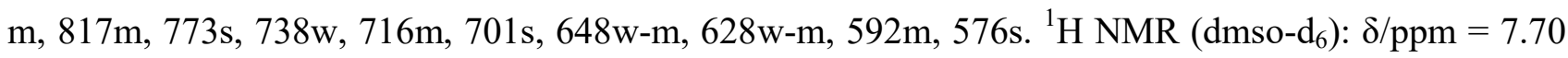
$\left(\mathrm{s}, 1 \mathrm{H}, \mathrm{C}^{2} \mathrm{H}\right) ; 7.56,7.36(\mathrm{~m}, 5 \mathrm{H}, \mathrm{Ph}) ; 4.57$ (s, $\left.5 \mathrm{H}, \mathrm{Cp}\right) ; 3.77,3.57$ (s, 6 H, NMe). ${ }^{13} \mathrm{C}\left\{{ }^{1} \mathrm{H}\right\} \mathrm{NMR}$ $\left(\mathrm{CDCl}_{3}\right): \delta / \mathrm{ppm}=270.0\left(\mathrm{CO}_{\text {acyl }}\right) ; 262.1\left(\mathrm{C}^{1}\right) ; 221.9(\mathrm{CO}) ; 169.5\left(\mathrm{C}^{3}\right) ; 146.3\left(\mathrm{C}^{2}\right) ; 132.8($ ipso-Ph); 129.1, $128.2(\mathrm{Ph}) ; 85.2(\mathrm{Cp}) ; 51.7,43.0(\mathrm{NMe})$. Crystallization from a $\mathrm{CH}_{2} \mathrm{Cl}_{2}$ solution layered with pentane and stored at $-30{ }^{\circ} \mathrm{C}$ afforded dark red crystals of $\mathbf{3 h}$. 
Chart 16. Structure of 3i.

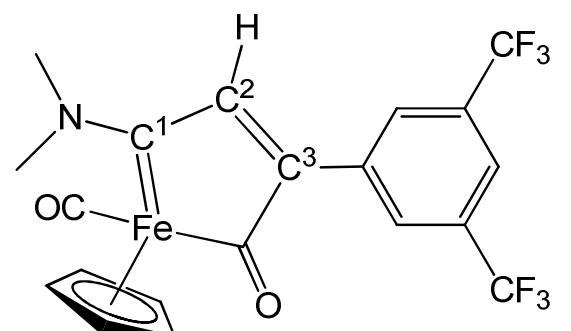

From $[\mathbf{2 i}] \mathrm{CF}_{3} \mathrm{SO}_{3}$. Brown solid, yield $45 \%$. Eluent for chromatography: $\mathrm{CH}_{2} \mathrm{Cl}_{2}$ (quick chromatography). Anal. calcd. for $\mathrm{C}_{20} \mathrm{H}_{15} \mathrm{~F}_{6} \mathrm{FeNO}_{2}$ : C, 50.98; H, 3.21; N, 2.97. Found: C, 51.15; H, 3.15; N, 2.94. IR $\left(\mathrm{CH}_{2} \mathrm{Cl}_{2}\right): \tilde{\mathrm{v}} / \mathrm{cm}^{-1}=1921 \mathrm{vs}(\mathrm{CO}), 1612 \mathrm{~m}\left(\mathrm{CO}_{\text {acyl }}\right), 1530 \mathrm{w}$. IR (solid state): $\tilde{\mathrm{v}} / \mathrm{cm}^{-1}=$ 2930w, 1908s (CO), 1608w-m (CO acyl $\left._{1}\right), 1594 \mathrm{w}-\mathrm{sh}, 1531 \mathrm{w}-\mathrm{m}, 1410 \mathrm{w}, 1400 \mathrm{w}, 1373 \mathrm{~m}-\mathrm{s}, 1276 \mathrm{vs}$, 1247w-m, 1169m-s, 1124vs, 1025s, 898w-m, 873w-m, 844m, 810m-s, 717w-m, 700m, 681s. ${ }^{1}$ H NMR $\left(\mathrm{dmso}_{6}\right): \delta / \mathrm{ppm}=8.33,8.25\left(\mathrm{~m}, 3 \mathrm{H}, \mathrm{C}_{6} \mathrm{H}_{3}\right) ; 8.05\left(\mathrm{~s}, 1 \mathrm{H}, \mathrm{C}^{2} \mathrm{H}\right) ; 4.58(\mathrm{~s}, 5 \mathrm{H}, \mathrm{Cp}) ; 3.71,3.67(\mathrm{~s}, 6 \mathrm{H}$, NMe). ${ }^{13} \mathrm{C}\left\{{ }^{1} \mathrm{H}\right\}$ NMR $\left(\right.$ dmso-d $\left._{6}\right): \delta / p p m=268.6\left(\mathrm{CO}_{\text {acyl }}\right) ; 256.6\left(\mathrm{C}^{1}\right) ; 222.8(\mathrm{CO}) ; 163.2\left(\mathrm{C}^{3}\right) ; 150.5$ $\left(\mathrm{C}^{2}\right) ; 135.3,130.0,125.2,130.5,122.3\left(\mathrm{~d},{ }^{2} \mathrm{~J}_{\mathrm{CF}}=32 \mathrm{~Hz}\right)$ (aromatics); $125.8\left(\mathrm{q},{ }^{1} \mathrm{~J}_{\mathrm{CF}}=418 \mathrm{~Hz}, \mathrm{CF}_{3}\right) ;$ 85.5 (Cp); 52.2, $44.7(\mathrm{NMe}) .{ }^{19} \mathrm{~F}\left\{{ }^{1} \mathrm{H}\right\} \mathrm{NMR}\left(\mathrm{dmso}_{\mathrm{d}}\right): \delta / \mathrm{ppm}=-61.3$.

\section{$\left[\mathrm{FeCp}(\mathrm{CO})\left\{\mathrm{C}^{1} \mathrm{~N}(\mathrm{Me})_{2} \mathrm{C}^{2} \mathrm{HC}{ }^{3}\left({ }^{\mathrm{t}} \mathrm{Bu}\right) \mathrm{C}(=\mathrm{O})\right\}\right], 3 \mathrm{j}(\mathrm{Chart} 17)$.}

\section{Chart 17. Structure of $\mathbf{3 j}$.}<smiles>CCCCC1=CC2C(CCC)=CC(C1=O)C21CC1</smiles> 
From $[\mathbf{2} \mathbf{j}] \mathrm{CF}_{3} \mathrm{SO}_{3}$. Brown solid, yield 43\%. Eluent for chromatography: $\mathrm{CH}_{2} \mathrm{Cl}_{2}$. Anal. calcd. for $\mathrm{C}_{16} \mathrm{H}_{21} \mathrm{FeNO}_{2}$ : C, 60.97; H, 6.72; N, 4.44. Found: C, 60.81; H, 6.80; N, 4.30. IR $\left(\mathrm{CH}_{2} \mathrm{Cl}_{2}\right): \tilde{v} / \mathrm{cm}^{-1}=$ 1909vs (CO), 1619m (CO $\left.\mathrm{CO}_{\text {acyl }}\right), 1528 \mathrm{w}$. IR (solid state): $\tilde{\mathrm{v}} / \mathrm{cm}^{-1}=2956 \mathrm{w}, 2867 \mathrm{w}, 1890 \mathrm{vs}(\mathrm{CO}), 1601 \mathrm{~s}$ $\left(\mathrm{CO}_{\text {acyl }}\right), 1541 \mathrm{~m}-\mathrm{s}, 1481 \mathrm{w}, 1455 \mathrm{w}, 1435 \mathrm{w}, 1407 \mathrm{~m}, 1387 \mathrm{w}, 1358 \mathrm{w}-\mathrm{m}, 1242 \mathrm{~m}, 1166 \mathrm{w}, 1098 \mathrm{w}, 1063 \mathrm{~m}-$ s, 1018w-m, 874w-m, 865m, 846m-s, 818m, 806m-s, 743m-s, 720w-m, 704m. ${ }^{1} \mathrm{H}$ NMR (dmso-d ${ }_{6}$ ): $\delta / \mathrm{ppm}=7.46\left(\mathrm{~s}, 1 \mathrm{H}, \mathrm{C}^{2} \mathrm{H}\right) ; 4.42(\mathrm{~s}, 5 \mathrm{H}, \mathrm{Cp}) ; 3.63,3.50(\mathrm{~s}, 6 \mathrm{H}, \mathrm{NMe}) ; 1.12\left(\mathrm{~s}, 9 \mathrm{H}, \mathrm{CMe}_{3}\right) .{ }^{13} \mathrm{C}\left\{{ }^{1} \mathrm{H}\right\}$ NMR (dmso-d 6$): \delta / p p m=270.2\left(\mathrm{CO}_{\text {acyl }}\right) ; 258.4\left(\mathrm{C}^{1}\right) ; 223.2(\mathrm{CO}) ; 182.7\left(\mathrm{C}^{3}\right) ; 146.2\left(\mathrm{C}^{2}\right) ; 85.3(\mathrm{Cp})$; 51.7, $43.6(\mathrm{NMe}) ; 34.4\left(\mathrm{CMe}_{3}\right) ; 29.3\left(\mathrm{CMe}_{3}\right)$.

$\left[\mathrm{FeCp}(\mathrm{CO})\left\{\mathrm{C}^{1} \mathrm{~N}(\mathrm{Me})_{2} \mathrm{C}^{2} \mathrm{HC}^{3}(2\right.\right.$-naphthyl)C(=O)\}], 3k (Chart 18).

Chart 18. Structure of $3 k$.

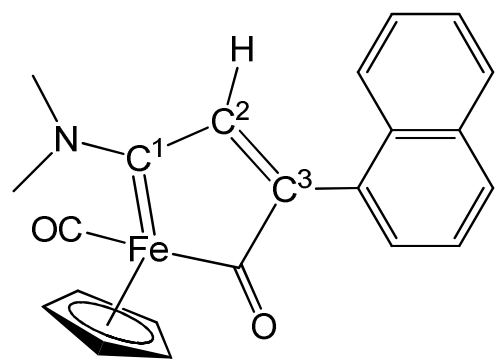

From $[\mathbf{2 k}] \mathrm{CF}_{3} \mathrm{SO}_{3}$. Brown solid, yield 48\%. Eluent for chromatography: $\mathrm{Et}_{2} \mathrm{O}$ (quick chromatography). Anal. calcd. for $\mathrm{C}_{22} \mathrm{H}_{19} \mathrm{FeNO}_{2}$ : C, 68.59; H, 4.97; N, 3.64. Found: C, 68.50; H, 5.06; N, 3.48. IR $\left(\mathrm{CH}_{2} \mathrm{Cl}_{2}\right): \tilde{v} / \mathrm{cm}^{-1}=1916 \mathrm{vs}(\mathrm{CO}), 1608 \mathrm{~m}\left(\mathrm{CO}_{\text {acyl }}\right), 1530 \mathrm{w}$. IR (solid state): $\tilde{\mathrm{v}} / \mathrm{cm}^{-1}=2925 \mathrm{w}, 2855 \mathrm{w}$, 1896vs (CO), 1604m (CO $\left.\mathrm{CO}_{\text {acyl }}\right), 1531 \mathrm{~m}, 1505 \mathrm{~m}, 1447 \mathrm{~m}, 1407 \mathrm{~m}, 1394 \mathrm{~m}, 1358 \mathrm{w}, 1331 \mathrm{w}, 1178 \mathrm{w}, 1152 \mathrm{~m}$, 1082s, 1051s, 963m-s, 917w, 864m-s, 843m-s, 800vs, 728m-s, 710s, 666m. ${ }^{1} \mathrm{H}$ NMR (dmso-d 6 ): $\delta / p p m$ $=7.94,7.53\left(\mathrm{~m}, 7 \mathrm{H}, \mathrm{C}_{10} \mathrm{H}_{7}\right) ; 7.21\left(\mathrm{~s}, 1 \mathrm{H}, \mathrm{C}^{2} \mathrm{H}\right) ; 4.63(\mathrm{~s}, 5 \mathrm{H}, \mathrm{Cp}) ; 3.74,3.58(\mathrm{~s}, 6 \mathrm{H}, \mathrm{NMe}) .{ }^{13} \mathrm{C}\left\{{ }^{1} \mathrm{H}\right\}$ NMR $\left(\mathrm{dmso}_{\mathrm{d}}\right): \delta / \mathrm{ppm}=266.9\left(\mathrm{CO}_{\text {acyl }}\right) ; 257.0\left(\mathrm{C}^{1}\right) ; 223.2(\mathrm{CO}) ; 171.0\left(\mathrm{C}^{3}\right) ; 151.3\left(\right.$ ipso- $\left.\mathrm{C}_{10} \mathrm{H}_{7}\right)$; $150.5\left(\mathrm{C}^{2}\right) ; 134.0,133.4,131.6,128.4,126.7,126.3,125.5\left(\mathrm{C}_{10} \mathrm{H}_{7}\right) ; 85.5(\mathrm{Cp}) ; 52.0,44.3(\mathrm{NMe})$. 
Chart 19. Structure of 31.

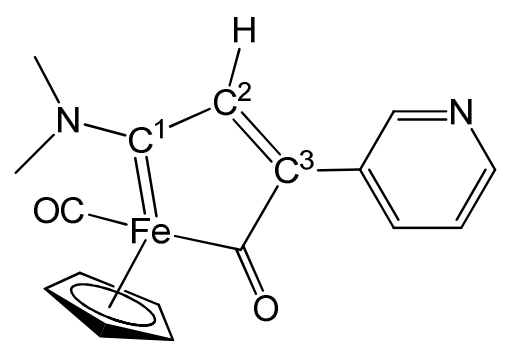

From $[2 \mathrm{2}] \mathrm{CF}_{3} \mathrm{SO}_{3}$. Brown solid, yield 55\%. Eluent for chromatography: $\mathrm{THF} / \mathrm{CH}_{2} \mathrm{Cl}_{2}$ 1:1 v/v (quick chromatography). Anal. calcd. for $\mathrm{C}_{17} \mathrm{H}_{16} \mathrm{FeN}_{2} \mathrm{O}_{2}: \mathrm{C}, 60.74 ; \mathrm{H}, 4.80 ; \mathrm{N}, 8.33$. Found: $\mathrm{C}, 60.54 ; \mathrm{H}, 4.81$; $\mathrm{N}, 8.25$. IR $\left(\mathrm{CH}_{2} \mathrm{Cl}_{2}\right): \tilde{v} / \mathrm{cm}^{-1}=1918 \mathrm{vs}(\mathrm{CO}), 1664 \mathrm{~m}-\mathrm{s}, 1604 \mathrm{~m}\left(\mathrm{CO}_{\text {acyl }}\right), 1528 \mathrm{w}-\mathrm{m}$. IR (solid state): $\tilde{\mathrm{v}} / \mathrm{cm}^{-1}=2981 \mathrm{~s}, 2971 \mathrm{~s}, 1885 \mathrm{vs}(\mathrm{CO}), 1604 \mathrm{~m}\left(\mathrm{CO}_{\mathrm{acyl}}\right), 1578 \mathrm{w}, 1560 \mathrm{w}, 1531 \mathrm{~m}, 1470 \mathrm{w}, 1405 \mathrm{w}-\mathrm{m}$, 1394w, 1237w, 1151w, 1085w, 1049w, 1026w-m, 994w-m, 881w, 810m, 784m, 712m, 702w-m. ${ }^{1} \mathrm{H}$ NMR $\left(\mathrm{dmso}^{\left.-\mathrm{d}_{6}\right)}\right) \delta / \mathrm{ppm}=8.74,8.54,7.93,7.41\left(\mathrm{~m}, 4 \mathrm{H}, \mathrm{C}_{5} \mathrm{H}_{4} \mathrm{~N}\right) ; 8.15\left(\mathrm{~s}, 1 \mathrm{H}, \mathrm{C}^{2} \mathrm{H}\right) ; 4.56(\mathrm{~s}, 5 \mathrm{H}, \mathrm{Cp})$; 3.70, $3.63(\mathrm{~s}, 6 \mathrm{H}, \mathrm{NMe}) .{ }^{13} \mathrm{C}\left\{{ }^{1} \mathrm{H}\right\} \mathrm{NMR}\left(\mathrm{dmso}_{6}\right): \delta / \mathrm{ppm}=268.6\left(\mathrm{CO}_{\text {acyl }}\right) ; 256.6\left(\mathrm{C}^{1}\right) ; 222.9(\mathrm{CO})$; $164.5\left(\mathrm{C}^{3}\right) ; 149.7\left(\mathrm{C}^{2}\right) ; 148.9,136.8,129.1,125.4,123.7\left(\mathrm{C}_{5} \mathrm{H}_{4} \mathrm{~N}\right) ; 85.5(\mathrm{Cp}) ; 52.0,44.3(\mathrm{NMe})$.

$\left[\mathrm{FeCp}(\mathrm{CO})\left\{\mathrm{C}^{1} \mathrm{~N}(\mathrm{Me})\left(\mathrm{CH}_{2} \mathrm{Ph}\right) \mathrm{C}^{2} \mathrm{HC}^{3}(\mathrm{Ph}) \mathrm{C}(=\mathrm{O})\right\}\right], 3 \mathrm{~m}(\mathrm{Chart} 20)$.

Chart 20. Structure of $\mathbf{3 m}$.

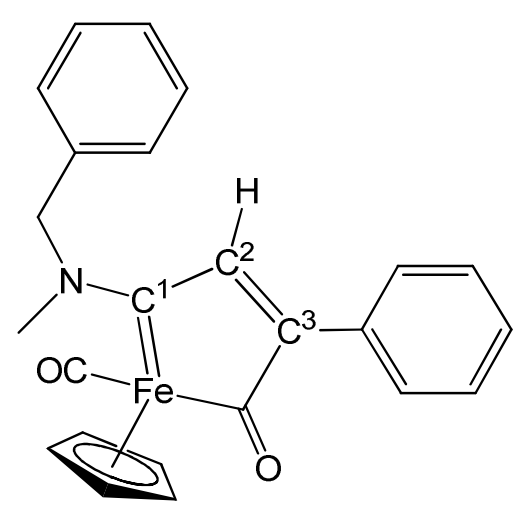


From $[\mathbf{2 m}] \mathrm{CF}_{3} \mathrm{SO}_{3}$. Brown solid, yield $80 \%$. Eluent for chromatography: $\mathrm{CH}_{2} \mathrm{Cl}_{2} / \mathrm{Et}_{2} \mathrm{O}$ 1:1 v/v. Anal. calcd. for $\mathrm{C}_{24} \mathrm{H}_{21} \mathrm{FeNO}_{2}$ : C, 70.09; H, 5.15; N, 3.41. Found: C, 70.31; H, 5.16; N, 3.50. IR $\left(\mathrm{CH}_{2} \mathrm{Cl}_{2}\right)$ : $\tilde{\mathrm{v}} / \mathrm{cm}^{-1}=1917 \mathrm{vs}(\mathrm{CO}), 1605 \mathrm{~m}\left(\mathrm{CO}_{\text {acyl }}\right), 1571 \mathrm{w}, 1509 \mathrm{~m}$. IR (solid state): $\tilde{\mathrm{v}} / \mathrm{cm}^{-1}=3029 \mathrm{vw}, 2929 \mathrm{vw}$, $1897 \mathrm{vs}(\mathrm{CO}), 1599 \mathrm{~m}\left(\mathrm{CO}_{\mathrm{acyl}}\right), 1569 \mathrm{w}, 1507 \mathrm{~m}, 1496 \mathrm{~m}, 1453 \mathrm{w}, 1443 \mathrm{w}, 1398 \mathrm{w}-\mathrm{m}, 1351 \mathrm{w}, 1242 \mathrm{w}$, 1201w, 1130w, 1090w-m, 1032w, 1005m, 996m, 970w, 871w, 842w, 812m, 773m-s, 735m, 720m, 695s. ${ }^{1} \mathrm{H}$ NMR $\left(\mathrm{CDCl}_{3}\right): \delta / \mathrm{ppm}=7.79,7.72\left(\mathrm{~s}, 1 \mathrm{H}, \mathrm{C}^{2} \mathrm{H}\right) ; 7.62-7.17\left(\mathrm{~m}, 10 \mathrm{H}, \mathrm{Ph}+\mathrm{CH}_{2} P h\right) ; 5.65$, $5.28\left(\mathrm{~d},{ }^{2} \mathrm{~J}_{\mathrm{HH}}=14.2 \mathrm{~Hz}, \mathrm{CH}_{2}\right) ; 5.20,5.06\left(\mathrm{~d}, 2 \mathrm{H},{ }^{2} \mathrm{~J}_{\mathrm{HH}}=15.8 \mathrm{~Hz}, \mathrm{CH}_{2}\right) ; 4.62,4.57(\mathrm{~s}, 5 \mathrm{H}, \mathrm{Cp}) ; 3.73$, $3.38(\mathrm{~s}, 3 \mathrm{H}, \mathrm{NMe})$. Isomer ratio $=\mathrm{ca} \cdot 1.2 .{ }^{13} \mathrm{C}\left\{{ }^{1} \mathrm{H}\right\} \mathrm{NMR}\left(\mathrm{CDCl}_{3}\right): \delta / \mathrm{ppm}=269.9\left(\mathrm{CO}_{\text {acyl }}\right) ; 264.4$, $264.0\left(\mathrm{C}^{1}\right)$; 222.1, $221.8(\mathrm{CO}) ; 170.3,169.9\left(\mathrm{C}^{3}\right) ; 146.6\left(\mathrm{C}^{2}\right) ; 134.7,134.4,132.8,132.7$ (ipso-Ph); 129.4, 129.3, 129.2, 128.4, 128.3, 128.2, 127.5, $126.4\left(\mathrm{Ph}+\mathrm{CH}_{2} \mathrm{Ph}\right) ; 85.4,85.2(\mathrm{Cp}) ;$ 67.9, 59.1 $\left(\mathrm{CH}_{2}\right)$; 49.9, $40.6(\mathrm{NMe})$.

\section{3) Synthesis and characterization of $\left[\mathrm{FeCp}(\mathrm{CO})\left\{\mathrm{C}^{1} \mathrm{NMe}(\mathrm{Xyl}) \mathrm{C}^{2} \mathrm{HC}^{3}(2-m e t h y l-\right.\right.$ pyridinium $) \mathrm{C}(=\mathrm{O})\}] \mathrm{CF}_{3} \mathrm{SO}_{3},[4] \mathrm{CF}_{3} \mathrm{SO}_{3}(\mathrm{Chart} 21)$.}

Chart 21. Structure of the cation of $[4] \mathrm{CF}_{3} \mathrm{SO}_{3}$.

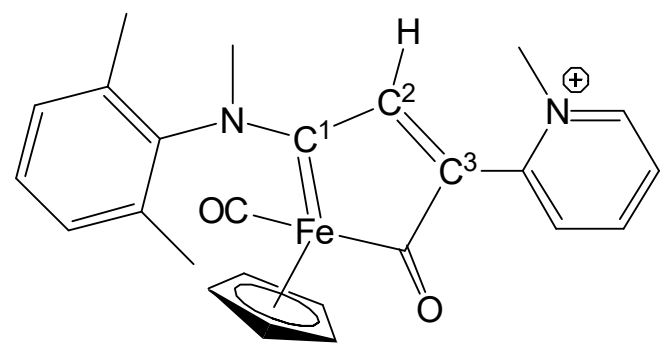

A solution of $\mathbf{3 d}(80 \mathrm{mg}, 0.188 \mathrm{mmol})$ in $\mathrm{CH}_{2} \mathrm{Cl}_{2}(10 \mathrm{~mL})$ was treated with methyl triflate $(0.025 \mathrm{~mL}$, $0.22 \mathrm{mmol}$ ). The resulting solution was allowed to stir at room temperature for 1 hour, then it was charged on a short alumina pad. Quick elution with $\mathrm{MeCN} / \mathrm{MeOH}$ mixture $(9: 1 \mathrm{v} / \mathrm{v})$ gave a brown fraction. The title product was obtained as a brown solid upon removal of the volatiles under vacuum. Yield 89 mg, 80\%. Anal. calcd. for $\mathrm{C}_{26} \mathrm{H}_{25} \mathrm{~F}_{3} \mathrm{FeN}_{2} \mathrm{O}_{5} \mathrm{~S}$ : C, 52.89; H, 4.27; N, 4.75. Found: C, 52.77; H, 
4.36; N, 4.85. IR $\left(\mathrm{CH}_{2} \mathrm{Cl}_{2}\right): \tilde{v} / \mathrm{cm}^{-1}=1939 \mathrm{vs}(\mathrm{CO}), 1610 \mathrm{~m}\left(\mathrm{CO}_{\text {acyl }}\right)$. IR (solid state): $\tilde{v} / \mathrm{cm}^{-1}=3553 \mathrm{w}$, 3490w, 3089w, 3067w, 2932vw, 1918s (CO), 1622m (COacyl), 1604m, 1587m, 1505m-s, 1483m, $1398 \mathrm{~m}, 1360 \mathrm{w}, 1277 \mathrm{~s}, 1252 \mathrm{vs}, 1226 \mathrm{~s}, 1154 \mathrm{vs}, 1087 \mathrm{~m}, 1030 \mathrm{vs}, 1010 \mathrm{~s}, 964 \mathrm{~m}, 893 \mathrm{w}-\mathrm{m}, 813 \mathrm{~m}-\mathrm{s}, 789 \mathrm{~s}$, 768s, $715 \mathrm{~m} .{ }^{1} \mathrm{H}$ NMR $\left(\mathrm{CD}_{2} \mathrm{Cl}_{2}\right): \delta / \mathrm{ppm}=8.84,8.29,7.90,7.52,7.29\left(\mathrm{~m}, 7 \mathrm{H}, \mathrm{C}_{5} \mathrm{H}_{4} \mathrm{~N}+\mathrm{C}_{6} \mathrm{H}_{3} \mathrm{Me}_{2}\right) ; 7.07$ (s, $\left.1 \mathrm{H}, \mathrm{C}^{2} \mathrm{H}\right) ; 4.83$ (s, $\left.5 \mathrm{H}, \mathrm{Cp}\right) ; 4.10,3.92(\mathrm{~s}, 6 \mathrm{H}, \mathrm{NMe}) ; 2.29,2.20\left(\mathrm{~s}, 6 \mathrm{H}, \mathrm{C}_{6} \mathrm{H}_{3} M e_{2}\right) .{ }^{13} \mathrm{C}\left\{{ }^{1} \mathrm{H}\right\} \mathrm{NMR}$ $\left(\mathrm{CD}_{2} \mathrm{Cl}_{2}\right): \delta / \mathrm{ppm}=264.2,263.2\left(\mathrm{CO}_{\text {acyl }}+\mathrm{C}^{1}\right) ; 220.2(\mathrm{CO}) ; 161.4\left(\mathrm{C}^{3}\right) ; 152.5\left(\mathrm{C}^{2}\right) ; 146.8,145.4,145.0$, 144.3, 132.0, 129.6, 129.3, 128.8, $126.9\left(\mathrm{C}_{5} \mathrm{H}_{4} \mathrm{~N}+\mathrm{C}_{6} \mathrm{H}_{3} \mathrm{Me}_{2}\right) ; 85.6(\mathrm{Cp}) ; 50.2,47.4(\mathrm{NMe}) ; 17.5,17.3$ $\left(\mathrm{C}_{6} \mathrm{H}_{3} M e_{2}\right)$. Crystals of $[4] \mathrm{CF}_{3} \mathrm{SO}_{3}$ suitable for $\mathrm{X}$-ray analysis were collected from a dichloromethane solution layered with hexane, stored at $-30{ }^{\circ} \mathrm{C}$.

\section{4) X-ray crystallography.}

Crystal data and collection details for $[\mathbf{2 i}] \mathrm{CF}_{3} \mathrm{SO}_{3},[\mathbf{2} \mathbf{j}] \mathrm{CF}_{3} \mathrm{SO}_{3}, \mathbf{3 b}, \mathbf{3 f}, \mathbf{3 g}, \mathbf{3 h}$ and $[\mathbf{4}] \mathrm{CF}_{3} \mathrm{SO}_{3} \cdot \mathrm{CH}_{2} \mathrm{Cl}_{2}$ are reported in Table 4. Data were recorded on a Bruker APEX II diffractometer equipped with a PHOTON100 detector using Mo-K $\alpha$ radiation. Data were corrected for Lorentz polarization and absorption effects (empirical absorption correction SADABS). ${ }^{[38]}$ The structures were solved by direct methods and refined by full-matrix least-squares based on all data using $F^{2}{ }^{[39]}$ Hydrogen atoms were fixed at calculated positions and refined by a riding model. All non-hydrogen atoms were refined with anisotropic displacement parameters.

Table 4. Crystal data and measurement details for $[\mathbf{2 i}] \mathrm{CF}_{3} \mathrm{SO}_{3},[\mathbf{2 j}] \mathrm{CF}_{3} \mathrm{SO}_{3} \cdot$ solv, $\mathbf{3 b}, \mathbf{3 f}, \mathbf{3 g}$, $\mathbf{3 h}$ and $[4] \mathrm{CF}_{3} \mathrm{SO}_{3} \cdot \mathrm{CH}_{2} \mathrm{Cl}_{2}$.

\begin{tabular}{lcccccccc}
\hline & {$[\mathbf{2 i}] \mathrm{CF}_{3} \mathrm{SO}_{3}$} & {$[2 \mathrm{j}] \mathrm{CF}_{3} \mathrm{SO}_{3} \cdot$ solv } & $\mathbf{3 b}$ & $\mathbf{3 f}$ & $\mathbf{3 g}$ & $\mathbf{3 h}$ & {$[\mathbf{4}] \mathrm{CF}_{3} \mathrm{SO}_{3} \cdot \mathrm{CH}_{2} \mathrm{Cl}_{2}$} \\
\hline Formula & $\mathrm{C}_{26} \mathrm{H}_{20} \mathrm{~F}_{9} \mathrm{Fe}_{2} \mathrm{NO}_{5} \mathrm{~S}_{22}$ & $\mathrm{C}_{22} \mathrm{H}_{26} \mathrm{~F}_{3} \mathrm{Fe}_{2} \mathrm{NO}_{5} \mathrm{~S}$ & $\mathrm{C}_{23} \mathrm{H}_{27} \mathrm{FeNO}_{2}$ & $\mathrm{C}_{23} \mathrm{H}_{21} \mathrm{FeNO}_{2} \mathrm{~S}$ & $\mathrm{C}_{24} \mathrm{H}_{20} \mathrm{ClFeNO}_{2}$ & $\mathrm{C}_{18} \mathrm{H}_{17} \mathrm{FeNO}_{2}$ & $\mathrm{C}_{27} \mathrm{H}_{27} \mathrm{FeCl}_{2} \mathrm{~N}_{2} \mathrm{O}_{2} \mathrm{~S}$ \\
$\mathrm{FW}$ & 741.19 & 585.20 & 405.30 & 431.32 & 445.71 & 335.17 & 675.31 \\
$\mathrm{~T}, \mathrm{~K}$ & $295(2)$ & $100(2)$ & $293(2)$ & $293(2)$ & $100(7)$ & $100(7)$ & $100(2)$ \\
$\lambda, \AA$ & 0.71073 & 0.71073 & 0.71073 & 0.71073 & 0.71073 & 0.71073 & 0.71073 \\
Crystal system & Monoclinic & Monoclinic & Monoclinic & Monoclinic & Monoclinic & Orthorhombic & Triclinic \\
Space group & $P 2_{1} / c$ & $P 2_{1} / n$ & $P 2_{1} / c$ & $C 2 / c$ & $C 2 / c$ & $F d d 2$ & $P \overline{1}$ \\
$a, \AA$ & $12.8947(8)$ & $7.8885(5)$ & $9.7227(10)$ & $17.6422(7)$ & $18.6544(13)$ & $26.648(3)$ & $8.1984(8))$ \\
$b, \AA$ & $12.1379(7)$ & $10.0925(7)$ & $23.904(2)$ & $11.5607(5))$ & $11.4297(8)$ & $28.827(3)$ & $8.9595(8)$
\end{tabular}




\begin{tabular}{|c|c|c|c|c|c|c|c|}
\hline$c, \AA$ & $18.0241(11)$ & $32.409(2)$ & $10.1857(10)$ & $20.0757(8)$ & $19.6644(14)$ & $7.8480(8)$ & $21.115(2)$ \\
\hline$\alpha,{ }^{\circ}$ & 90 & 90 & 90 & 90 & 90 & 90 & $80.077(3)$ \\
\hline$\beta,^{\circ}$ & $96.610(2)$ & $95.162(2)$ & $117.240(3)$ & $96.7890(10)$ & $99.767(2)$ & 90 & $84.622(3)$ \\
\hline$\gamma^{\circ}$ & 90 & 90 & 90 & 90 & 90 & 90 & $66.656(3)$ \\
\hline Cell Volume, $\AA^{3}$ & $2802.3(3)$ & $2569.8(3)$ & 2104.8(4) & $4065.9(3)$ & $4132.0(5)$ & $6028.8(11)$ & $1402.1(2)$ \\
\hline Z & 4 & 4 & 4 & 8 & 8 & 16 & 2 \\
\hline$D_{c}, \mathrm{~g} \cdot \mathrm{cm}^{-3}$ & 1.757 & 1.513 & 1.279 & 1.409 & 1.433 & 1.477 & 1.600 \\
\hline$\mu, \mathrm{mm}^{-1}$ & 1.209 & 1.264 & 0.733 & 0.863 & 0.879 & 1.006 & 0.865 \\
\hline$F(000)$ & 1488 & 1200 & 856 & 1792 & 1840 & 2784 & 692 \\
\hline Crystal size, mm & $0.22 \times 0.19 \times 0.14$ & $0.21 \times 0.16 \times 0.13$ & $0.25 \times 0.21 \times 0.14$ & $0.16 \times 0.14 \times 0.11$ & $0.24 \times 0.21 \times 0.14$ & $0.18 \times 0.16 \times 0.12$ & $0.22 \times 0.18 \times 0.14$ \\
\hline$\theta$ limits, $^{\circ}$ & $1.590-27.101$ & 2.114-26.994 & $1.704-25.998$ & $2.043-24.998$ & 2.098-26.998 & $2.081-26.997$ & $1.959-27.997$ \\
\hline $\begin{array}{l}\text { Reflections } \\
\text { collected }\end{array}$ & 38900 & 33066 & 25609 & 24198 & 28059 & 16704 & 20270 \\
\hline $\begin{array}{l}\text { Independent } \\
\text { reflections }\end{array}$ & $\begin{array}{c}6182\left[R_{\text {int }}=\right. \\
0.0403]\end{array}$ & $\begin{array}{c}5608\left[R_{\text {int }}=\right. \\
0.0521]\end{array}$ & $\begin{array}{c}4119\left[R_{\text {int }}=\right. \\
0.0981]\end{array}$ & $\begin{array}{c}3577\left[R_{\text {int }}=\right. \\
0.0417]\end{array}$ & $\begin{array}{c}4498\left[R_{\text {int }}=\right. \\
0.0332]\end{array}$ & $\begin{array}{c}3265\left[R_{\text {int }}=\right. \\
0.0768]\end{array}$ & $\begin{array}{c}6718\left[R_{\text {int }}=\right. \\
0.0378]\end{array}$ \\
\hline $\begin{array}{l}\text { Data / restraints } \\
\text { /parameters }\end{array}$ & $6182 / 318 / 453$ & $5608 / 0 / 312$ & $\begin{array}{c}4119 / 162 \text { / } \\
278\end{array}$ & $\begin{array}{c}3577 / 211 / \\
269\end{array}$ & 4498 / 4 / 270 & 3265 / 67 / 202 & $6718 / 0 / 374$ \\
\hline $\begin{array}{l}\text { Goodness on fit } \\
\text { on } \mathrm{F}^{2}\end{array}$ & 1.047 & 1.294 & 1.144 & 1.103 & 1.200 & 1.185 & 1.144 \\
\hline$R_{1}(I>2 \sigma(I))$ & 0.0565 & 0.0727 & 0.0902 & 0.0413 & 0.0444 & 0.0656 & 0.0537 \\
\hline$w R_{2}$ (all data) & 0.1487 & 0.1442 & 0.1804 & 0.0969 & 0.0923 & 0.1226 & 0.1021 \\
\hline $\begin{array}{l}\text { Largest diff. } \\
\text { peak and hole, e } \\
\AA^{-3}\end{array}$ & $1.129 /-0.533$ & $0.990 /-0.831$ & $0.804 /-0.351$ & $0.302 /-0.258$ & $0.451 /-0.464$ & $0.986 /-1.043$ & $0.804 /-0.686$ \\
\hline
\end{tabular}




\section{5) Stability studies.}

a) Stability in $\mathrm{D}_{2} \mathrm{O} /$ dmso- $_{6}$. Each compound (3b-m and $\left.[4] \mathrm{CF}_{3} \mathrm{SO}_{3}, c a .3 \mathrm{mg}\right)$ was dissolved in dmso$\mathrm{d}_{6} / \mathrm{D}_{2} \mathrm{O}($ ca. $3: 1 \mathrm{v} / \mathrm{v})$. The resulting solutions were analyzed by ${ }^{1} \mathrm{H}$ NMR and then maintained at $37{ }^{\circ} \mathrm{C}$ for $72 \mathrm{~h}$. After cooling to room temperature, the final solutions were analyzed by ${ }^{1} \mathrm{H}$ NMR: the resonances of the starting compound were clearly recognized, with no significant variations (total amount of other species $<10 \%$ respect to the starting compound).

b) Stability in cell culture medium. Each compound (3b-m and $[4] \mathrm{CF}_{3} \mathrm{SO}_{3}$, ca. $\left.6 \mathrm{mg}\right)$ was dissolved in dmso (ca. $4 \mathrm{~mL}$ ) in a glass tube, then $2 \mathrm{~mL}$ of RPMI-1640 medium (Merck; modified with sodium bicarbonate, without L-glutamine and phenol red, liquid, sterile-filtered, suitable for cell culture) were added. The resulting mixture was maintained at $37{ }^{\circ} \mathrm{C}$ for $72 \mathrm{~h}$, then allowed to cool to room temperature and extracted with $\mathrm{CH}_{2} \mathrm{Cl}_{2}(5-10 \mathrm{~mL})$. Removal of the volatiles from the organic phase gave a residue (3-4 $\mathrm{mg}$ ) which was analyzed by IR spectroscopy $\left(\mathrm{CH}_{2} \mathrm{Cl}_{2}\right.$ solution), the IR spectrum being superimposable with that of the starting material.

\section{6) Electrochemistry.}

Cyclic voltammograms were measured in a $\mathrm{N}_{2}$ glovebox (MBRAUN LABmaster) with levels of $\mathrm{H}_{2} \mathrm{O}$ and $\mathrm{O}_{2}$ below 0.1 ppm using a Gamry Interface $1000 \mathrm{~b}$ potentiostat controlled by Gamry Framework software. Solvents were dried and distilled under Ar from the appropriate drying agent (THF from $\mathrm{Na} / \mathrm{K}$ and benzophenone, $\mathrm{MeCN}$ from $\mathrm{CaH}_{2}$ ), stored over $3 \AA$ molecular sieves and thoroughly deoxygenated with Ar prior use. The samples were measured in acetonitrile or tetrahydrofuran at a concentration of $1 \mathrm{mM}$ of complex and $0.1 \mathrm{M}$ of $\mathrm{Bu}_{4} \mathrm{NPF}_{6}$ as conductive salt. A glassy carbon electrode was used as working electrode, a platinum disk as counter electrode and a silver wire as a pseudoreference electrode. Ferrocene (or decamethylferrocene) was added as an internal standard and all spectra were referenced to the ferrocene/ferrocenium redox couple $\left(\mathrm{Fc} / \mathrm{Fc}^{+}\right)$. 


\section{7) In vitro cytotoxicity study.}

Human ovarian carcinoma (A2780 and A2780cisR) cell lines were obtained from the European Collection of Cell Cultures. The human embryonic kidney (HEK-293) cell line was obtained from ATCC (Sigma, Buchs, Switzerland). Penicillin streptomycin, RPMI 1640 GlutaMAX (where RPMI = Roswell Park Memorial Institute), and DMEM GlutaMAX media (where DMEM = Dulbecco's modified Eagle medium) were obtained from Life Technologies, and fetal bovine serum (FBS) was obtained from Sigma. The cells were cultured in RPMI 1640 GlutaMAX (A2780 and A2780cisR) and DMEM GlutaMAX (HEK-293) media containing 10\% heat-inactivated FBS and 1\% penicillin streptomycin at $37{ }^{\circ} \mathrm{C}$ and $\mathrm{CO}_{2}(5 \%)$. The A2780cisR cell line was routinely treated with cisplatin $(2$ $\mu \mathrm{M})$ in the media to maintain cisplatin resistance. The cytotoxicity was determined using the 3-(4,5dimethyl 2-thiazolyl)-2,5-diphenyl-2H-tetrazolium bromide (MTT) assay. ${ }^{40}$ Cells were seeded in flatbottomed 96-well plates as a suspension in a prepared medium $(100 \mu \mathrm{L}$ aliquots and approximately 4300 cells/well) and preincubated for $24 \mathrm{~h}$. Stock solutions of compounds were prepared in dmso and were diluted in medium. The solutions were sequentially diluted to give a final dmso concentration of $0.5 \%$ and a final compound concentration range $(0-200 \mu \mathrm{M})$. Cisplatin and RAPTA-C were tested as a positive $(0-100 \mu \mathrm{M})$ and negative $(200 \mu \mathrm{M})$ controls respectively. The compounds were added to the preincubated 96-well plates in $100 \mu \mathrm{L}$ aliquots, and the plates were incubated for a further $72 \mathrm{~h}$. MTT (20 $\mu \mathrm{L}, 5 \mathrm{mg} / \mathrm{mL}$ in Dulbecco's phosphate buffered saline) was added to the cells, and the plates were incubated for a further $4 \mathrm{~h}$. The culture medium was aspirated and the purple formazan crystals, formed by the mitochondrial dehydrogenase activity of vital cells, were dissolved in dmso (100 $\mu \mathrm{L} / \mathrm{well})$. The absorbance of the resulting solutions, directly proportional to the number of surviving cells, was quantified at $590 \mathrm{~nm}$ using a SpectroMax M5e multimode microplate reader (using SoftMax Pro software, version 6.2.2). The percentage of surviving cells was calculated from the absorbance of wells 
corresponding to the untreated control cells. The reported $\mathrm{IC}_{50}$ values are based on the means from two independent experiments, each comprising four tests per concentration level.

\section{Acknowledgements}

We gratefully thank the University of Pisa for financial support (PRA_2017_25, "composti di metalli di transizione come possibili agenti antitumorali”) and the Swiss National Science Foundation for financial support.

\section{Supporting Information Available}

Views of X-ray structures (Figures S1-S2); NMR spectra (Figures S3-S43); cyclic voltammograms (Figures S44-S56). CCDC reference numbers $1944506\left([\mathbf{2 i}] \mathrm{CF}_{3} \mathrm{SO}_{3}\right), 1944507\left([\mathbf{2 j}] \mathrm{CF}_{3} \mathrm{SO}_{3}\right), 1944508$ (3b), 1944509 (3f), $1944510(\mathbf{3 g}), 1944511(\mathbf{3 h})$ and $1944512\left([\mathbf{4}] \mathrm{CF}_{3} \mathrm{SO}_{3}\right)$ contain the supplementary crystallographic data for the X-ray studies reported in this paper. These data can be obtained free of charge at www.ccdc.cam.ac.uk/conts/retrieving.html (or from the Cambridge Crystallographic Data Centre, 12, Union Road, Cambridge CB2 1EZ, UK; fax: (internat.) +44-1223/336-033; e-mail: deposit@ccdc.cam.ac.uk).

\section{References}

1 (a) I. Bauer, H. J. Knolker, Chem. Rev. 2015, 115, 3170-3387. (b) A. Fürstner, ACS Cent. Sci. 2016, 2, 778789. (c) Topics in Organometallic Chemistry 50, Iron Catalysis II, ed. Springer, Ed. E. Bauer, 2016. (d) S. Enthaler, K. Junge, M. Beller, Angew. Chem. Int. Ed. 2008, 47, 3317-3321.

2 (a) C. Johnson, M. Albrecht, Coord. Chem. Rev. 2017, 352, 1-14. (b) C. Johnson, M. Albrecht, Organometallics 2017, 36, 2902-2913. (c) T. Liu, D. L. DuBois, R. M. Bullock, Nat. Chem. 2013, 5, 228233. (d) M. D. Bala, M. I. Ikhile, J. Mol. Catal. A 2014, 385, 98-105.

3 (a) M. Patra, G. Gasser, Nat. Rev. Chem. 2017, 1, 1-12. (b) G. Jaouen, A. Vessiéres, S. Top, Chem. Soc. Rev. 2015, 44, 8802-8817. (c) M. Patra, G. Gasser, M. Wenzel, K. Merz, J. E. Bandow, N. Metzler-Nolte, Organometallics 2012, 31, 5760-5771. 
4 (a) A. Pilon, P. Gírio, G. Nogueira, F. Avecilla, H. Adams, J. Lorenzo, M. H. Garcia, A. Valente, J. Organomet. Chem. 2017, 852, 34-42. (b) P. R. Florindo, D. M. Pereira, P. M. Borralho, C. M. P. Rodrigues, M. F. M. Piedade, A. C. Fernandes, J. Med. Chem. 2015, 58, 4339-4347. (c) W. A. Wani, U. Baig, S. Shreaz, R. A. Shiekh, P. F. Iqbal, E. Jameel, A. Ahmad, S. H. Mohd-Setapar, M. Mushtaque, L. Ting Hun, New J. Chem. 2016, 40, 1063-1090. (d) A. Valente, A. M. Santos, L. Côrte-Real, M. P. Robalo, V. Moreno, M. Font-Bardia, T. Calvet, J. Lorenzo, M. H. Garcia, J. Organomet. Chem. 2014, 756, 52-60. (e) A. C. Gonçalves, T. S. Morais, M. P. Robalo, F. Marques, F. Avecilla, C. P. Matos, I. Santos, A. I. Tomaz, M. H. Garcia, J. Inorg. Biochem. 2013, 129, 1-8.

5 X. Jiang, L. Chen, X. Wang, L. Long, Z. Xiao, X. Liu, Chem. Eur. J. 2015, 21, 13065-13072.

6 N. J. Coville, E. A. Darling, A. W. Hearn, P. Johnston, J. Organomet. Chem. 1987, 328, 375-385.

7 W. E. Williams, F. J. Lalor, J. Chem. Soc., Dalton Trans. 1973, 13, 1329-1332.

8 See for instance: (a) A. P. Prakasham, M. K. Gangwar, P. B. Ghosh, Eur. J. Inorg. Chem. 2019, 295-313. (b) J. Ruiz, L. García, M. Vivanco, D. Sol, S. García-Granda, Dalton Trans. 2017, 46, 10387-10398. (c) S. Yasuda, H. Yorimitsu, K. Oshima, Organometallics 2008, 27, 4025-4027.

9 (a) A. R. Manning, G. McNally, J. Organomet. Chem. 1988, 338, 383-392. (b) R. Kumar, A. R. Manning, P. T. Murray, J. Organomet. Chem. 1987, 323, 53-65.

10 R. J. Angelici, J. W. Dunker, Inorg. Chem. 1985, 24, 2209-2215.

11 C. P. Casey, S. R. Marder, R. E. Colborn, P. A. Goodson, Organometallics 1986, 5, 199-203.

12 (a) E. J. Crawford, T. W. Bodnar, A. R. Cutler, J. Am. Chem. Soc. 1986, 108, 6202-6212. (b) L. H. Gade, H. Memmler, U. Kauper, A. Schneider, S. Fabre, I. Bezougli, M. Lutz, C. Galka, I. J. Scowen, M. McPartlin, Chem. Eur. J. 2000, 6, 692-708.

13 (a) S. El-Tarhuni,; L. M. Manhaes, C. Morrill, J. Raftery, J. K. Randhawa, M. W. Whiteley, J. Organomet. Chem. 2016, 811, 20-25. (b) D. J. Crowther, Z. Zhang, G. J. Palenik, W. M. Jones, Organometallics 1992, 11, 622-628. (c) H. Adams, N. A. Bailey, M. Grayson, C. Ridgway, A. J. Smith, P. Taylor, M. Winter, Organometallics 1990, 9, 2621-2628. (d) H. Adams, C. A. Maloney, J. E. Muir, S. J. Walters, M. J. Winter, J. Chem. Soc., Chem. Commun. 1995, 1511-1512. (e) L.Busetto, C. Camiletti, V. Zanotti, V. G. Albano, P. Sabatino, J. Organomet. Chem. 2000, 593-594, 335-341.

14 (a) I. Yu, C. J. Wallis, B. O. Patrick, P. L. Diaconescu, P. Mehrkhodavandi, Organometallics 2010, 29, 6065-6076. (b) S. G. Davies, A. J. Edwards, S. Jones, M. R. Metzler, K. Yanada, R. Yanada, J. Chem. Soc., Dalton Trans. 1998, 1587-1594. (c) H. Adams, N. A. Bailey, C. Ridgway, B. F. Taylor, S. J. Walters, M. J. J. Organomet. Chem. 1990, 394, 349-364. (d) J. Park, J. Kim, Organometallics 1995, 14, 4431-4434.

15 S. G. Eaves, D. S. Yufit, B. W. Skelton, J. A. K. Howard, P. J. Low, Dalton Trans. 2015, 44, 14341-14348.

16 R. D. Adams, D. F. Chodosh, N. M. Golembeski, E. C. Weissman, J. Organomet. Chem. 1979, 172, 251267. 
17 (a) F. Marchetti, Eur. J. Inorg. Chem. 2018, 3987-4003, and references therein. (b) R. Mazzoni, M. Salmi, V. Zanotti, Chem. Eur. J. 2012, 18, 10174-10194. (c) P. Lang, M. Schwalbe, Chem. Eur. J. 2017, 23, 17398-17412. (d) B. S. Natinsky, C. Liu, Nat. Chem. 2019, 11, 199-203. (e) P. Tong, D. Yang, Y. Li, B. Wang, J. Qu, Organometallics 2015, 34, 3571-3576. (f) W.-S. Ojo, F. Y. Pétillon, P. Schollhammer, J. Talarmin, Organometallics 2008, 27, 4207-4222. (g) R. Mazzoni, F. Marchetti, A. Cingolani, V. Zanotti, Inorganics 2019, 7, 25.

18 G. Agonigi, M. Bortoluzzi, F. Marchetti, G. Pampaloni, S. Zacchini, V. Zanotti, Eur. J. Inorg. Chem. 2018, 960-971.

19 (a) V. G. Albano, L. Busetto, F. Marchetti, M. Monari, S. Zacchini, V. Zanotti, Organometallics 2003, 22, 1326-1331. (b) G. Ciancaleoni, S. Zacchini, V. Zanotti, F. Marchetti, Organometallics 2018, 37, $3718-3731$.

20 D. Rocco, L. K. Batchelor, G. Agonigi, S. Braccini, F. Chiellini, S. Schoch, T. Biver, T. Funaioli, S. Zacchini, L. Biancalana, M. Ruggeri, G. Pampaloni, P. J. Dyson, F. Marchetti, Chem. Eur. J., DOI 10.1002/chem. 201902885.

21 See for instance: (a) F. Marchetti, S. Zacchini, V. Zanotti, Organometallics 2018, 37, 107-115. (b) L. Busetto, F. Marchetti, S. Zacchini, V. Zanotti, Organometallics 2006, 25, 4808-4816. (c) V. G. Albano, L. Busetto, F. Marchetti, M. Monari, S. Zacchini, V. Zanotti, Organometallics 2004, 23, 3348-3354. (d) V. G. Albano, L. Busetto, F. Marchetti, M. Monari, S. Zacchini, V. Zanotti, J. Organomet. Chem. 2006, 691, 4234-4243.

22 G. Agonigi, G. Ciancaleoni, T. Funaioli, S. Zacchini, F. Pineider, C. Pinzino,; G. Pampaloni, V. Zanotti, F. Marchetti, Inorg. Chem. 2018, 57, 15172-15186.

23 L. Busetto, F. Marchetti, S. Zacchini, V. Zanotti, Organometallics 2005, 24, 2297-2306.

24 (a) J. Hu, J. Wang, T. H. Nguyen, N. Zheng, Beilstein J. Org. Chem. 2013, 9, 1977-2001. (b) N. G. Connelly, W. E. Geiger, Chem. Rev. 1996, 96, 877-910. (c) M. Bortoluzzi, F. Marchetti, G. Pampaloni, S. Zacchini, Inorg. Chem. 2014, 53, 3832-3838. (d) M. Periasamy, G. Srinivas, P. Bharathi, J. Org. Chem. 1999, 64, 4204-4205.

25 T.-aki Mitsudo, K.-ichi Fujita, S. Nagano, T.-aki Suzuki, Y. Watanabe, H. Masuda, Organometallics 1995, $14,4228-4235$.

26 F. H. Allen, O. Kennard, D. G. Watson, L. Brammer, A. G. Orpen, R. Taylor, J. Chem. Soc. Perkin Trans. II 1987, $\mathrm{S} 1-\mathrm{S} 19$.

27 S. G. Eaves, D. S. Yufit, B. W. Skelton, J. A. K. Howard, P. J. Low, Dalton Trans. 2015, 44, 14341-14348.

28 (a) Z. Lamprecht, N. A. van Jaarsveld, D. I. Bezuidenhout, D. C. Liles, S. Lotz, Dalton Trans. 2015, 44, 19218-19231. (b) B. Nandi, S. Sinha, Tetrahedron 2011, 67, 106-113. (c) L. Busetto, F. Marchetti, S. Zacchini, V. Zanotti, Eur. J. Inorg. Chem. 2005, 3250-3260.

29 A. J. Bard, L. R. Faulkner, Electrochemical Methods, Ed. Wiley \& Sons, Inc., Hoboken NJ, $2^{\text {nd }}$ Ed., 2001. 
30 B. S. Murray, M. V. Babak, C. G. Hartinger, P. J. Dyson, Coord. Chem. Rev. 2016, 306, 86-114, and references therein.

31 S. Pathania, R. K. Narang, R. K. Rawal, Eur. J. Med. Chem. 2019, 180, 486-508.

32 Y. Gothe, T. Marzo, L. Messori, N. Metzler-Nolte, Chem. Eur. J. 2016, 22, 12487-12494.

33 (a) D. Gibson, Dalton Trans. 2016, 45, 12983-12991. (b) C. G. Hartinger, M. A. Jakupec, S. ZorbasSeifried, M. Groessl, A. Egger, W. Berger, H. Zorbas, P. J. Dyson, B. K. Keppler, Chem. Biodivers. 2008, 5, 2140-2154, and references therein. (c) R. G. Kenny, C. J. Marmion, Chem. Rev. 2019, 119, 1058-1137.

34 (a) C. G. Hartinger, A. D. Phillips, A. A. Nazarov, Curr. Top. Med. Chem. 2011, 11, 2688-2702. (b) J. Furrer, G. Süss-Fink, Coord. Chem. Rev. 2016, 309, 36-50. (c) M. V. Babak, W. H. Ang, Met. Ions Life Sci. 2018, 18, 171-198. (d) L. K. Batchelor, P. J. Dyson, Trends in Chemistry 2019, 1, 644-655.

35 A. Nguyen, A. Vessières, E. A. Hillard, S. Top, P. Pigeon, G. Jaouen, Chimia 2007, 61, 716-724.

36 G. R. Fulmer, A. J. M. Miller, N. H. Sherden, H. E. Gottlieb, A. Nudelman, B. M. Stoltz, J. E. Bercaw, K. I. Goldberg, Organometallics 2010, 29, 2176-2179.

37 W. Willker, D. Leibfritz, R. Kerssebaum, W. Bermel, Magn. Reson. Chem. 1993, 31, 287-292.

38 G. M. Sheldrick, SADABS-2008/1 - Bruker AXS Area Detector Scaling and Absorption Correction, Bruker AXS: Madison, Wisconsin, USA, 2008.

39 G. M. Sheldrick, Acta Crystallogr. C 2015, 71, 3-8.

40 T. Mosmann, J. Immunol. Methods 1983, 65, 55-63. 
